\title{
Matrix architecture plays a pivotal role in 3D osteoblast migration: the effect of interstitial fluid flow
}

\author{
Cristina Del Amo ${ }^{1,2}$, Vanesa Olivares ${ }^{1,2}$, Mar Cóndor ${ }^{1,2}$, Alejandro Blanco ${ }^{1,3}$, Jorge Santolaria ${ }^{2,3}$, \\ Jesús Asín ${ }^{4}$, Carlos Borau ${ }^{1,2}$, José Manuel García-Aznar ${ }^{1,2}$ \\ 1. Multiscale in Mechanical and Biological Engineering, Department of Mechanical Engineering, University of Zaragoza, Zaragoza, Spain \\ 2. Aragon Institute of Engineering Research, University of Zaragoza, Zaragoza, Spain \\ 3. Department of Design and Manufacturing Engineering, University of Zaragoza, Zaragoza, Spain \\ 4. Department of Statistical Methods, University of Zaragoza, Zaragoza, Spain
}

*Corresponding author

Prof. J. Manuel García-Aznar. Mechanical Engineering Department. Betancourt Building - Rio Ebro Campus - University of Zaragoza. María de Luna s/n. 50018 Zaragoza (Spain) E -mail: jmgaraz@unizar.es Tel: +34 976762 796. Fax: +34 976762670

\begin{abstract}
Osteoblast migration is a crucial process in bone regeneration, which is strongly regulated by interstitial fluid flow. However, the exact role that such flow exerts on osteoblast migration is still unclear. To deepen the understanding of this phenomenon, we cultured human osteoblasts on 3D microfluidic devices under different fluid flow regimes. Our results show that a slow fluid flow rate by itself is not able to alter the 3D migratory patterns of osteoblasts in collagen-based gels but that at higher fluid flow rates (increased flow velocity) may indirectly influence cell movement by altering the collagen microstructure. In fact, we observed that high fluid flow rates $(1 \mu \mathrm{l} / \mathrm{min})$ are able to alter the collagen matrix architecture and to indirectly modulate the migration pattern. However, when these collagen scaffolds were crosslinked with a chemical crosslinker, specifically, transglutaminase II, we did not find significant alterations in the scaffold architecture or in osteoblast movement. Therefore, our data suggest that high interstitial fluid flow rates can regulate osteoblast migration by means of modifying the orientation of collagen fibers. Together, these results highlight the crucial role of the matrix architecture in 3D osteoblast migration. In addition, we show that interstitial fluid flow in conjunction with the matrix architecture regulates the osteoblast morphology in 3D.
\end{abstract}

Competing Interests: A provisional Spanish patent application that covers the support for the fluid flow application disclosed in this manuscript has been submitted. CDA, AB, JS, and JMGA are listed as inventors on this application.

Keywords: osteoblast migration, transglutaminase, interstitial fluid flow, fibers, collagen-based matrices, crosslinkers

\section{Introduction}

Fracture healing is a complex biological process that restores structural bone integrity [1,2]; the mechanochemical microenvironment regulates all the steps in this process, from sequential tissue morphogenesis to a cascade of cellular and molecular events. The ability of bone to self-repair involves the coordination of cellular and mechanosensitive processes, especially cell differentiation, migration and surveillance [3-5]. This complex process is regulated by multiple microenvironmental factors that effectively determine the

This is the post-print version of the following article: Del Amo, C., Olivares, V., Cóndor, M., Blanco, A., Santolaria, J., Asín, J., Borau C \& García-Aznar, J. M. (2018). Matrix architecture plays a pivotal role in 3D osteoblast migration: The effect of interstitial fluid flow. Journal of the mechanical behavior of biomedical materials, 83, 52-62. doi: 10.1016/j.jmbbm.2018.04.007, which has been published in final form here. 
outcome of bone fracture healing. To unravel this complexity, experimental methodologies and computational modeling have been widely performed and combined [6-10] to improve the knowledge of this regenerative process.

The success of the fracture healing requires the achievement of some specific regenerative patterns that involve the coordination of multiple cellular events like cell proliferation, differentiation and migration of different cell types [11]. Although most of fractures heal, there are conditions that complicate bone healing [12] such as the increase of the elderly population or the proliferation of diseases like diabetes. Current treatment of non-union bone fractures usually involves surgery to stabilize the area and the insertion of a bone graft at the site to stimulate vasculogenesis and osteogenesis (the formation of new bone). In this work, we aim to improve the knowledge on bone healing. Specifically, we investigate how micro environmental factors may regulate cell migration during the bone healing process. During fracture healing, bone formation requires mature osteoblasts to deposit bone with remarkable spatial precision [13]. Therefore, osteoblasts must migrate within the three-dimensional matrix space in order to get to their destination, where bone will be deposited [14]. Hence, most of computational models [15] that simulate bone fracture healing incorporate cell migration as a fundamental process to regulate the final outcome of the fracture. A better knowledge of how micro environmental factors can mediate this osteoblast migration capacity could improve the treatment of bone fractures, although we have to keep in mind that osteoblast migration is only one of the processes that are combined and work simultaneously with other events and with other interactive cell types.

Among all the microenvironmental factors that are involved in fracture healing, mechanics plays the most important role [16], and it is normally associated with the stability of the fracture gap. Indeed, primary fracture healing occurs when the fracture gap presents extreme stability while secondary healing implies moderately stability, occurring in the vast majority of bone injuries [1,6]. Recently, several tissue differentiation theories have been proposed, and they have been virtually tested by computers using different mechanistic-based stimuli, such as strain, pore pressure or fluid flow [17-21]. The theory of Lacroix and Prendergast [7] proposed an algorithm to predict the evolution of intramembranous and endochondral ossification, assuming that precursor cells can differentiate into the corresponding specialized tissue cells influenced by the effect of mechanical variables, namely, the deviatoric strain and the interstitial fluid flow velocity. Similarly, the theory of Gómez-Benito et al. [18] proposes a similar assumption but only considering the deviatoric strain. In a more recent work, Gonzalez-Torres et al. [17] demonstrated the relevant role of interstitial fluid flow in tissue differentiation under cyclic loading conditions. There are really few in-vitro experiments that analyze the role of interstitial fluid flow on osteoblast cells, because most of these works are based on the application of direct fluid flow in a parallel plate flow chamber [22,23]. Some of these works analyzed the cell response to understand the cell mechanisms of adaptation to mechanical stimulus such as fluid flow (abbreviated FF) [22,24-27]. In this context, their results showed a rapid increase ( $2-4$ fold) in the nitric oxide or prostaglandin levels released by osteocytes when FF was applied, compared to the response of osteoblasts and preosteoblasts. Moreover, compared to static conditions, the release of signaling molecules such as cyclic adenosine monophosphate (cAMP) or prostaglandin E2 (PGE2) was increased in responses to fluid shear stress [28].

The role of the extracellular matrix (ECM) is also critical for osteoblast migration. Via focal adhesions, osteoblasts recognize the ECM by adhering to it and by modifying their behavior depending on the matrix component or the mechano-chemical stimuli applied [13]. Actually, type I collagen is the most abundant bone matrix protein $[29,30]$. The stiffness of the ECM in the damaged area increases during the stages from callus formation through collagen type I secretion to final bone, thus altering cell behavior during the bone

This is the post-print version of the following article: Del Amo, C., Olivares, V., Cóndor, M., Blanco, A., Santolaria, J., Asín, J., Borau C \& García-Aznar, J. M. (2018). Matrix architecture plays a pivotal role in 3D osteoblast migration: The effect of interstitial fluid flow. Journal of the mechanical behavior of biomedical materials, 83, 52-62. doi: 10.1016/j.jmbbm.2018.04.007, which has been published in final form here. 
healing process $[14,31,32]$. As a method to investigate osteoblast mechanotransduction, the substrate of the cell culture can be modified by using crosslinking methods (such as chemical crosslinkers), consequently varying the stiffness experienced by cells [24].

Other micro environmental factors, such as transforming growth factor beta (TGF- $\beta$ ) superfamily, bone morphogenetic proteins (BMPs), and interleukins (IL-6, IL17F, and IL1 $\beta$ ), can also play a regulatory role in bone fracture healing $[11,33,34]$. In addition, 3D experimental assays revealed a low chemotactic effect of growth factors on osteoblasts in terms of migration compared to the response of other cell types under similar conditions [35,36]. For instance, while fibroblasts exhibit directional migration under PDGF-BB gradients [37], osteoblast migration seems to be regulated by the ability of cells to remodel the matrix [35].

Despite the numerous 3D-culture systems and techniques already published, there are still few studies of the biomechanical response of bone cells. There are even less studies specifically about human primary osteoblasts embedded in 3D in vitro microenvironments since most of the bone studies have been developed with non-human cells, such as murine osteoblasts [38-40]. Previous studies have shown that both matrix characteristics (in terms of chemical composition) and fluid flow are important regulators of osteoblast behavior [41]. To the best of our knowledge, the effect of these factors on osteoblast migration has not been experimentally explored yet. Hence, the main aim of this work is to understand the role of interstitial fluid flow and how extracellular matrix variations affect osteoblast migration in 3D. For this purpose, we developed a microfluidic device that allows the application of interstitial fluid flow on collagen-based gels and allows high-quality image acquisition. This system allows quantifying not only migration parameters but also the fiber alignment and matrix porosity of collagen hydrogels. While keeping the collagen density constant, the microstructure was altered by adding the crosslinking enzyme transglutaminase 2 (TG2) [42]. TG2 is a multifunctional enzyme with $\mathrm{Ca}^{+2}$-dependent protein crosslinking activity that also exhibits GTPase, cell adhesion, protein disulfide isomerase, kinase and scaffold activities [43]. This molecule is involved in apoptosis, matrix stabilization and cell adhesion in a variety of tissues, with possible involvement in bone matrix maturation and calcification [44].

In this work, we quantify osteoblast migration patterns and compare them for different collagen-based architectures that were modified by varying the fluid flow conditions and using, or not, crosslinkers.

\section{Material and Methods}

\subsection{Fluid flow setup}

The geometry of the microfluidic devices used in the fluid flow system consists of a microfluidic device lodged inside a hand-made platform fabricated by rapid prototyping and precision mechanics. The components were connected with PTFE (polytetrafluoroethylene) tubing (inner $\varnothing=0.3 \mathrm{~mm}$, outer $\varnothing=0.6$ $\mathrm{mm}$ ) through connectors [Y tube fitting PVDF (polyvinylidene fluoride), male and female luer locks and screwed connectors M6] to two syringe pumps (Harvard Apparatus Pump 11 Elite, Harvard Apparatus. Massachusetts (United States)) (See Figure 1).

The geometry of the microfluidic devices used in these experiments was adapted from the chips developed by Farahat et al. (2012) with fabrication methodology $[45,46]$. The new design is based on a central chamber where a hydrogel is inserted to mimic the extracellular matrix. This chamber is surrounded by two channels that allow the application and control of different fluid flow conditions. The microfluidic geometry was patterned in an SU-8 wafer by soft lithography techniques, and this wafer served as a mold to cure the PDMS (poly(dimethlysiloxane)) (Sylgard 184, Dow Corning GmbH) in a 10:1 ratio) and to obtain the

This is the post-print version of the following article: Del Amo, C., Olivares, V., Cóndor, M., Blanco, A., Santolaria, J., Asín, J., Borau C \& García-Aznar, J. M. (2018). Matrix architecture plays a pivotal role in 3D osteoblast migration: The effect of interstitial fluid flow. Journal of the mechanical behavior of biomedical materials, 83, 52-62. doi: 10.1016/j.jmbbm.2018.04.007, which has been published in final form here. 
desired design. Plasma treatment was used for the irreversibly bonding of the PDMS device with a glass coverslip followed by coating with PDL (poly-D-lysine hydrobromide, $1 \mathrm{mg} / \mathrm{ml}$, Sigma) to enhance hydrogel adhesion. Trapezoidal posts delimit the central chamber also providing support for the confinement of the hydrogel. During the flow application, the fluid passes from one channel to another through the pores left by the fibers of the collagen gel. Therefore, fluid can only pass through the collagen. On the other hand, in relation to the cellular distribution in the gel, during the preparation of the collagen gel, the cell suspension was added to the mixture in order to achieve homogeneity. This mixture of collagen and cells was introduced into the central chamber for study. The height of the chip $(320 \mu \mathrm{m})$ allowed us to find cells completely embedded within the 3D gel.

Based on previous works, the initial pumping flow rate applied to the cell culture in order to simulate the interstitial fluid flow was $0.1 \mu \mathrm{l} / \mathrm{min}$ [47], followed later by the application of a higher pumping flow rate $(1 \mu \mathrm{l} / \mathrm{min})$ in order to increase the generated shear stress. Throughout this article, the flow rate refers to the pumping flow rate. The corresponding surface velocity for each of the values specified above was $1.5 \mu \mathrm{m} / \mathrm{s}$ for $0.1 \mu \mathrm{l} / \mathrm{min}$ and $15 \mu \mathrm{m} / \mathrm{s}$ for $1 \mu \mathrm{l} / \mathrm{min}$ pumping flow rate. Additionally, $1 \mu \mathrm{l} / \mathrm{min}$ was the flow rate applied in experiments for analyzing the effect of fluid flow on the matrix architecture. Figure 1A describes the geometry of this chip, the disposition of the collagen-based hydrogel onto the chip (located in the central chamber) and the flow direction.

To apply fluid flow in a systematic way, we developed a flow adapter consisting of a base with two holders, where the microfluidic device was hosted in touch with the microscope objective, and their corresponding lids, with holes to allow the connector input (see Figure 1B).

This is the post-print version of the following article: Del Amo, C., Olivares, V., Cóndor, M., Blanco, A., Santolaria, J., Asín, J., Borau C \& García-Aznar, J. M. (2018). Matrix architecture plays a pivotal role in 3D osteoblast migration: The effect of interstitial fluid flow. Journal of the mechanical behavior of biomedical materials, 83, 52-62. doi: 10.1016/j.jmbbm.2018.04.007, which has been published in final form here. 


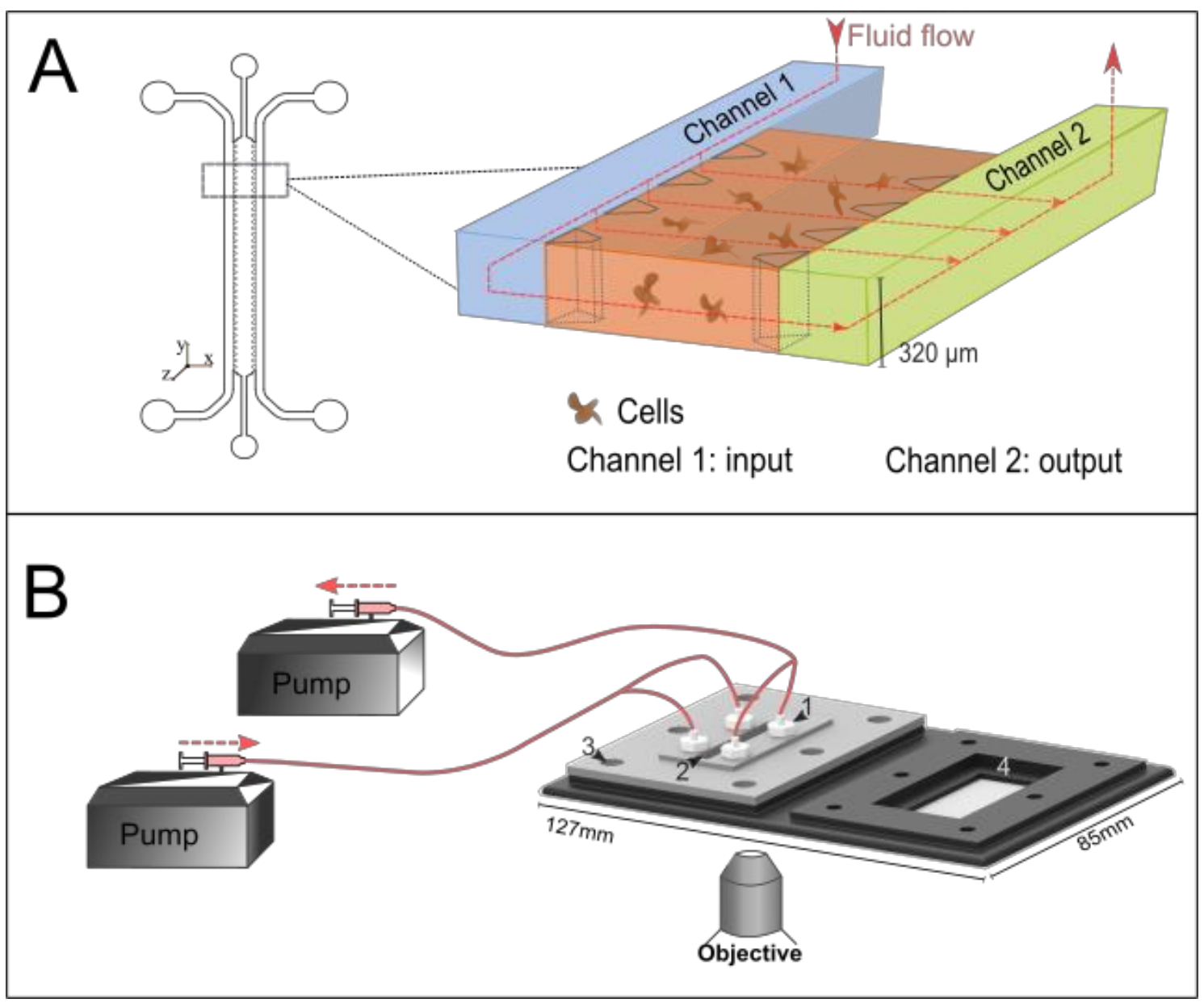

Figure 1. A) The general conformation of the microfluidic device and its transversal section to visualize the distribution of channels. B) A representative diagram of the fluid flow setup (1: input and output connectors; 2: microscopic visualization area; 3 : screw holes for the locking system; 4: recess for microfluidic chip insertion).

The base, fabricated in aluminum, consists of two compartments. Each compartment was approximately three millimeters larger than the PDMS devices to be fitted and had a window that allows microscopic observation of the microfluidic channels.

Covers were fabricated by means of additive manufacturing. The basis of this method is the production of parts shaped gradually and the addition of solid material [48], giving the user high versatility in terms of adaptable designs and chip geometries. They were designed to obtain a leak-proof system, and the cover elements are described below (see the corresponding element for each number in Figure 1). In the upper part of the cover, the following parts were distinguished: 1) four holes where the corresponding connectors of inputs and outputs of tubes are inserted, 2) a window to allow the passage of light during visualization of samples under the microscope, and 3) six holes for the locking system (six M3 screws and hexagonal nuts). The lower part of the lid, in contact with the chip, features the following elements: 4) a system that facilitates the user to fit the chip and 5) four recesses for the placement of O-rings (silicon, inner diameter $=4 \mathrm{~mm}$, thickness $=1 \mathrm{~mm}$; not shown in the Figure), which ensure the leak tightness of the system and

This is the post-print version of the following article: Del Amo, C., Olivares, V., Cóndor, M., Blanco, A., Santolaria, J., Asín, J., Borau C \& García-Aznar, J. M. (2018). Matrix architecture plays a pivotal role in 3D osteoblast migration: The effect of interstitial fluid flow. Journal of the mechanical behavior of biomedical materials, 83, 52-62. doi: 10.1016/j.jmbbm.2018.04.007, which has been published in final form here. 
which coincide with the holes punched in the microfluidic chip. The diameter of these holes is 3 times larger than the diameter of the chip holes.

Once the prototype of the encapsulation was defined, the covers were manufactured by the 3D Object Eden $350 \mathrm{~V}$ printer with the material RGD525, which was found to be the most appropriate material for the conditions to which the system was to be subjected (see S1-Supplementary Material). This platform has been provisionally covered by patent P201730809, property of the University of Zaragoza.

\subsection{D cell culture and hydrogel preparation}

Human osteoblast cells (HOBs; PromoCell) were used at low passages in these experiments, and osteoblast growth medium (PromoCell) was used as the cell culture medium. In total, $40 \mu l$ of cell suspension at a density of $1 \times 10^{6}$ cells $/ \mathrm{ml}$ was added to the collagen hydrogel by pipetting it into the central channel.

Collagen hydrogel type I (rat tail, BD Biosciences) was used at a final concentration of $4 \mathrm{mg} / \mathrm{ml}$ with DPBS $10 \mathrm{X}$ (Lonza), adjusting the $\mathrm{pH}$ to 7.4 with $0.5 \mathrm{M} \mathrm{NaOH}$. In hydrogels crosslinked with transglutaminase 2 (rhTGM2, R\&D Systems), this enzyme was added at the concentration of $25 \mu \mathrm{g} / \mathrm{ml}$ [35]. The polymerization of hydrogels and cell maintenance were performed in an incubator at $37^{\circ} \mathrm{C}$ and $5 \% \mathrm{CO} 2$ under humidity conditions, avoiding prepolymerization of the hydrogels.

Cells seeded in the collagen hydrogels were placed into the microfluidic devices at day 0 and kept in the incubator for $24 \mathrm{~h}$. Then, these devices were set in the support to apply fluid flow monitoring the experiment under the microscope. After approximately $24 \mathrm{~h}$ of fluid flow, the devices were taken from the fluid-flow support and fixed with 4\% PFA for immunostaining. A diagram of the different conditions and hydrogels used throughout this article has been attached to the Supplementary Material (S2).

Mechanical and microstructural characterization of $4 \mathrm{mg} / \mathrm{ml}$ collagen hydrogels was performed in a previous work by using a Haake Rheostress 1 rheometer for quantification of the hydrogel mechanical properties and a scanning electron microscope (SEM) for microarchitecture visualization (see table 1) [35].

\begin{tabular}{|c|c|c|c|c|}
\hline $\begin{array}{c}\text { Hydrogel } \\
\text { composition }\end{array}$ & $\begin{array}{c}\text { Storage } \\
\text { shear } \\
\text { modulus G' } \\
(\mathrm{Pa})\end{array}$ & $\begin{array}{c}\text { Maximum } \\
\text { shear strain }\end{array}$ & $\begin{array}{c}\text { Maximum } \\
\text { G' }(\mathrm{Pa})\end{array}$ & $\begin{array}{c}\text { Strain at } \\
\text { maximum G }\end{array}$ \\
\hline $4 \mathrm{mg} / \mathrm{ml}$ & $\begin{array}{c}121.03 \pm \\
9.94\end{array}$ & 0.403 & 191.2 & 0.403 \\
\hline $\begin{array}{c}4 \mathrm{mg} / \mathrm{ml} \\
+\mathrm{TG} 2\end{array}$ & $\begin{array}{c}127.90 \pm \\
14.43\end{array}$ & 0.449 & 285.5 & 0.384 \\
\hline
\end{tabular}

Table 1. Mechanical properties of the collagen hydrogels used for the in vitro experiments. Three samples were analyzed for the cases without TG2 and $25 \mu \mathrm{g} / \mathrm{ml}$ of TG2. Data taken with permission from [35].

\subsection{Image-based quantification of hydrogel architecture}

DQ-Collagen ${ }^{\mathrm{TM}}$ type I from bovine skin fluorescein conjugate (Thermo Fisher) was used to analyze the disposition of collagen fibers inside the hydrogel. The fluorescence of this reactant was used to determine the morphology of our hydrogels using a Nikon D-Eclipse C1 confocal microscope equipped with a 40X oil objective. In total, $10 \mu 1$ of DQ-Collagen ${ }^{\mathrm{TM}}$ was added to the collagen hydrogel (using a previously

This is the post-print version of the following article: Del Amo, C., Olivares, V., Cóndor, M., Blanco, A., Santolaria, J., Asín, J., Borau C \& García-Aznar, J. M. (2018). Matrix architecture plays a pivotal role in 3D osteoblast migration: The effect of interstitial fluid flow. Journal of the mechanical behavior of biomedical materials, 83, 52-62. doi: 10.1016/j.jmbbm.2018.04.007, which has been published in final form here. 
described procedure), obtaining a final concentration of $25 \mu \mathrm{g} / \mathrm{ml}$ [49], and softly pipetted into the microfluidic device. Then, samples were incubated overnight in an incubator and connected to the fluid system. The flow rate applied to the corresponding devices was $1 \mu 1 / \mathrm{min}$. Images were captured before and after the flow application to analyze the possible effects caused by this mechanical stimulus.

To the best of our knowledge, this is the first time this fluorescent reactant was used for this purpose since it is normally used for measuring matrix degradation by cells [49]. Colocalization of matrix images from confocal reflection, collagen immunostaining and DQ images was performed by analyzing the ratio of detected and overlapping fibers, thus confirming the validity of this technique (see Supplementary Material, S3).

Immunostaining for collagen was also performed to confirm our hypothesis about fiber distribution. The samples were incubated with monoclonal anti-collagen type I (mouse IgG isotype) (Sigma-Aldrich, C2456) overnight at $4^{\circ} \mathrm{C}$ after the samples were fixed with $4 \%$ paraformaldehyde (PFA, Affymetrix) in PBS for 15 $\mathrm{min}$ at room temperature based on the protocol proposed by Sung et al. 2009 [50]. Following blocking with serum and washing the channels, Alexa Fluor ${ }^{\circledR} 546$ donkey anti-mouse IgG $(\mathrm{H}+\mathrm{L})$ (Molecular Probes, A10036) was added, and the samples were incubated overnight at $4^{\circ} \mathrm{C}$.

Confocal z-stack images were sequentially acquired at one point at the center of each microdevice, maintaining a constant step size of $0.5 \mu \mathrm{m}$ and a maximum pixel dwell time. The 3D reconstruction of the cross-sectional collagen network images captured with a 40X oil objective (46 slides) was carried out by 3D skeletonization using the Ct-FIRE algorithm [51,52] and a binary stack was obtained (see Figure 2). Furthermore, we quantified the fiber orientation and pore size with another existing method [53]. Orientation was then obtained by considering the binary matrix as a distribution of masses. The tensor of inertia for each individual fiber was calculated, and the axis of minimum inertia, which points in the direction of the fiber, was obtained through diagonalization. Pore size was evaluated by obtaining the distribution of nearest obstacle distances (NODs), defined as the Euclidean distance from a point in the liquid phase to the nearest point in the solid phase.
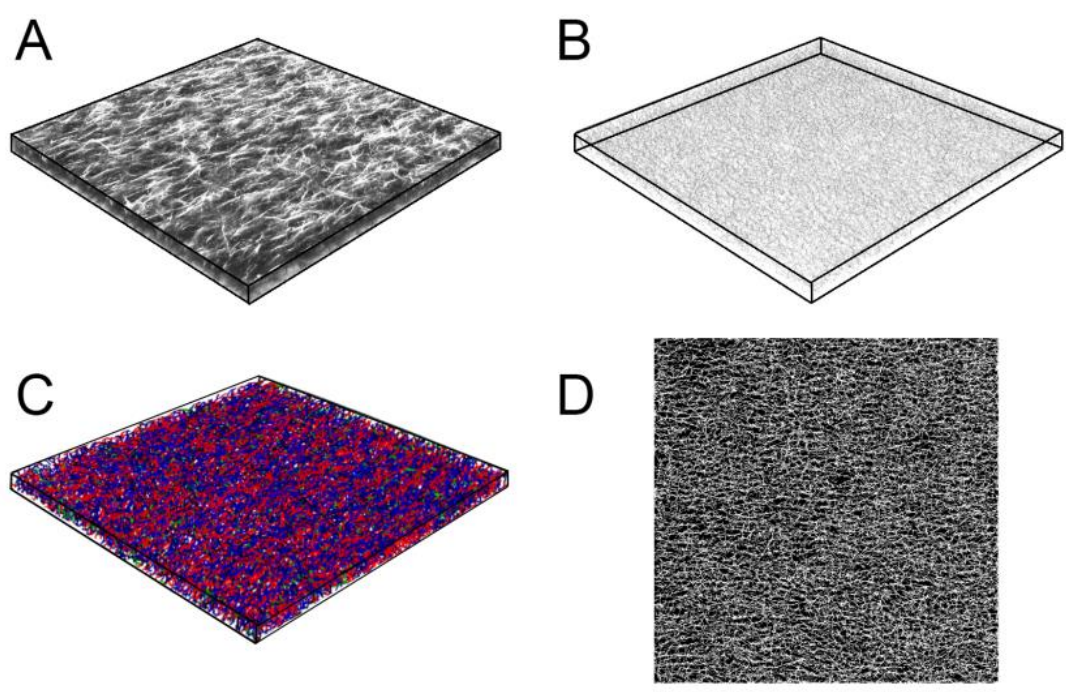

Figure 2. A) Stack of confocal cross-sections. B) Binarized stack visualized with Paraview. C) Ct-Fire reconstruction of the collagen network viewed in MATLAB. Color scale depending on orientation is added for a better visualization D) $2 \mathrm{D}$ projection of the obtained skeleton of a collagen matrix.

This is the post-print version of the following article: Del Amo, C., Olivares, V., Cóndor, M., Blanco, A., Santolaria, J., Asín, J., Borau C \& García-Aznar, J. M. (2018). Matrix architecture plays a pivotal role in 3D osteoblast migration: The effect of interstitial fluid flow. Journal of the mechanical behavior of biomedical materials, 83, 52-62. doi: 10.1016/j.jmbbm.2018.04.007, which has been published in final form here. 


\subsection{Image acquisition and cell tracking processing}

The complete system with the corresponding samples and hardware for the flow application were inserted into the incubator chamber of the microscope (Nikon D-Eclipse C1, 10X objective), and the samples were maintained at $37^{\circ} \mathrm{C}, 5 \% \mathrm{CO} 2$ and $95 \%$ humidity. Then, time-lapse imaging was performed by choosing the focal plane in the middle along the z-axis and by acquiring phase contrast images every 20 min for $24 \mathrm{~h}$. To track the cell trajectory, a hand-coded semi-automatic script implemented in MATLAB, already used in previous works, was employed [35-37]. These trajectories were used to extract cell mean (Vmean) and effective (Veff) velocities. Note that Vmean stands for the averaged instantaneous speed including all time steps, whereas Veff only takes into account the initial and final positions. MSD curve of each trajectory was also obtained and used to determine the global diffusion coefficient $(D)$ through linear weighted fit of the mean MSD curve (using the first quarter of the data). Also, MSD individual curves were used to fit a power law $\left.\left(\operatorname{MSD}(t)=\gamma \cdot t^{\alpha}\right)\right)$ to determine the kind of motion $(\alpha<1$ for confined motion, $\alpha=1$ for Brownian or purely diffusive motion and $\alpha>1$ for directed motion) [54].

ANOVA and Kruskal-Wallis tests were performed to assess statistical significance among the cell migration data sets, and statistical significance was assumed when $\mathrm{p}<0.001(* * *), \mathrm{p}<0.01(* *)$ or $\mathrm{p}<$ $0.05(*)$.

\subsection{Immunofluorescence and quantification of cell morphology}

Cells arranged within the collagen fibrillar network were fixed and immunostained by vinculin and phalloidin to assess focal adhesion formation and cytoskeletal distribution. Samples were fixed by incubation with 4\% paraformaldehyde for $20 \mathrm{~min}$ and then washed five times with PBS at room temperature (RT). Permeabilization of the cells was performed using $0.1 \%$ Triton X-100 (Calbiochem) in PBS for 10 min at RT. The cells were washed another three times and blocked with 5\% BSA/PBS (Sigma) with 3\% goat serum for $4 \mathrm{~h}$ at RT. Afterwards, mouse anti-human hVin-1 antibody (ab11194, Abcam) at 1:100 dilution in $0.5 \% \mathrm{BSA} / \mathrm{PBS}$ was added to the device and then incubated overnight at $4{ }^{\circ} \mathrm{C}$. After the device was washed with $0.5 \%$ BSA/PBS at least 5 times, it was incubated with Alexa Fluor ${ }^{\circledR} 488$ goat anti-mouse antibody (A11029, Molecular Probes) at 1:100 dilution and with phalloidin-tetramethylrhodamine B isothicyanate (TRITC) (Sigma-Aldrich P1951) at 1:200 dilution for $3 \mathrm{~h}$ at RT in the dark, followed by three washes with $0.5 \%$ BSA/PBS and subsequent washes with PBS alone. Finally, DAPI (Invitrogen, D1306) was added at a ratio of 1:50 to stain the cell nuclei, and the samples were incubated for $1 \mathrm{~h}$ in the dark at RT. Then, the samples were washed again with $0.5 \%$ BSA/PBS and subsequently imaged using an Olympus Fluoview FV10i confocal microscope equipped with an UPLSAPO 60XW objective.

Additionally, quantitative analysis of the cell morphology these immunostained samples was performed by using a Nikon D-Eclipse C1 confocal microscope (40X-oil objective) to acquire images and a hand-coded script implemented in MATLAB to process them. Two main parameters were measured: cell solidity and cell major axis length. The former is calculated as the cell area divided by the area of the minimum convex polygon containing the cell. This solidity value helps to measure the irregularity or star-like shape of cells. Small values correspond to cells with thin bodies and long spread arms, and higher values relate to either spread or thin cells without protrusions (see Figure 6D).

This is the post-print version of the following article: Del Amo, C., Olivares, V., Cóndor, M., Blanco, A., Santolaria, J., Asín, J., Borau C \& García-Aznar, J. M. (2018). Matrix architecture plays a pivotal role in 3D osteoblast migration: The effect of interstitial fluid flow. Journal of the mechanical behavior of biomedical materials, 83, 52-62. doi: 10.1016/j.jmbbm.2018.04.007, which has been published in final form here. 


\subsection{Quantification of interstitial fluid flow in microfluidic devices}

Throughout this work, the flow rate value refers to that introduced into the pump programing. However, to determine the distribution and velocity of the fluid flow inside the hydrogel, two complementary methodologies were proposed. On the one hand, real fluid velocity was obtained by tracking fluorescent particles through the hydrogel, whereas computational simulations estimated this speed and the distribution of fluid along the channels and the central chamber. In this section, both approaches are explained and their corresponding results are summarized in the Supplementary Material (see section S4).

\subsubsection{Bead tracking}

Carboxilated beads (FluoSpheres ${ }^{\mathrm{TM}}$ Carboxylate-Modified Microspheres, $0.2 \mu \mathrm{m}$, red fluorescent (580/605)-, Life Technologies F8887), which were previously sonicated, were used to quantify the flow velocities into the hydrogel. A hand-coded script was implemented in MATLAB to track the beads and obtain their velocity. To quantify the movement of beads into the collagen hydrogel, the concentration of gel selected to perform this experiment was $1.5 \mathrm{mg} / \mathrm{ml}$ in order to simplify the experimental setup. Images were captured every second during a 5 min period with a fluorescence microscope (Nikon D-Eclipse C1, Plan Fluor ELWD 40x Ph2 ADL). The distribution of fluid flow inside the $4 \mathrm{mg} / \mathrm{ml}$ hydrogel was simulated and compared to the results obtained in this experimental assay.

\subsubsection{Computational model}

Computational fluid dynamics was used to predict the flow rate inside the chamber using COMSOL Multiphysics 4.3 finite-element code (COMSOL AB). The model represents the geometry with a central channel, which lodges the hydrogel and the channels from each reservoir (see Figure 1A). The cell culture medium -assumed to be water- was defined as incompressible and homogenous, with a dynamic viscosity of $1 \times 10 \mathrm{E}-3 \mathrm{~Pa} \cdot \mathrm{s}$ (common for both hydrogels). The corresponding permeability for each gel concentration was $3.19 \times 10 \mathrm{E}-13 \mathrm{~m}^{2}$ for the $1.5 \mathrm{mg} / \mathrm{ml}$ hydrogel and $3.82 \times 10 \mathrm{E}-13 \mathrm{~m}^{2}$ for the $4 \mathrm{mg} / \mathrm{ml}$ hydrogel and was experimentally calculated following a procedure published in a previous work [55].

Two inlets were defined at the entries of the left channel and the contact area between the hydrogel and the channel. The same boundary conditions were imposed for the outlets in the opposite location of the right channel. PDMS surfaces were considered impermeable walls.

The device was divided in three regions: zones 1 and 3 correspond to the lateral channels, and zone 2 corresponds to the middle channel (see Figure 1A; zone 1 is in blue; zone 2, in orange; and zone 3, in green). In zone 2, the inertial term was neglected following the Stokes-Brinkman equation for incompressible flow. Laminar flow was used, however, for lateral channels. Extremely fine elements were used to mesh the relevant geometry. The size of the elements ranged between $8.19 \mathrm{E}-4 \mathrm{~mm}$ and $0.143 \mathrm{~mm}$, and the complete mesh consisted of 86802 elements.

\section{Results}

Several conditions were tested to explore the effect that extracellular matrix architecture and interstitial fluid flow exerts on 3D human osteoblast migration.

3.1 The application of $1 \mu \mathrm{l} / \mathrm{min}$ flow alters the architecture of non-crosslinked collagen-based gels

This is the post-print version of the following article: Del Amo, C., Olivares, V., Cóndor, M., Blanco, A., Santolaria, J., Asín, J., Borau C \& García-Aznar, J. M. (2018). Matrix architecture plays a pivotal role in 3D osteoblast migration: The effect of interstitial fluid flow. Journal of the mechanical behavior of biomedical materials, 83, 52-62. doi: 10.1016/j.jmbbm.2018.04.007, which has been published in final form here. 
The collagen network architecture was modified by applying a rapid fluid flow but was unaffected at low flow rates or when the network was crosslinked with TG2. This suggests that high flow rates are capable of dragging the collagen fibers, altering both the network orientation and pore size. Microscopic images were captured before and after the application of fluid flow and then analyzed following the methodology described in the Material and methods in subsection 2.3. Experimental conditions were divided into four cases: 1) collagen hydrogel, 2) collagen $+1 \mu \mathrm{l} / \mathrm{min}$ of fluid flow (FF), 3) collagen reticulated with TG2 (collagen + TG2), and 4) collagen $+\mathrm{TG} 2+1 \mu \mathrm{l} / \mathrm{min} \mathrm{FF}$. Three different experiments were performed for each condition $(\mathrm{n}=3)$. Note that in 1$)$ and 3$)$, a null flow rate was used.

To quantify the fiber orientation in 3D, a particular reference coordinate system represented by a semisphere was followed in this analysis (Figure 3A). Hydrogel charge into the microfluidic chamber was performed by small holes following the $\mathrm{Y}$ direction (axis 3), whereas the fluid movement crossed the hydrogel parallel to the $\mathrm{X}$ direction (axis 1). The $\mathrm{Z}$ direction represents the height of the channel (axis 13). After the reconstruction of a 3D stack, the network structure was extracted to obtain fiber alignment, porosity and pore size. To visualize fiber distribution, all the main directions of the fibers were classified into angular sectors (3D cones) whose representative vectors are shown in Fig. 3A. Fig. 3B shows a 3D rose diagram, scaled according to the number of fibers and normalized among all cases, revealing stronger alignment of the network mainly in the $\mathrm{X}$ direction and less strong alignment in the $\mathrm{Z}$ direction (axes 1 and 13 , respectively) for all studied cases. Alignment in $\mathrm{Z}$ is probably due to gravity during polymerization. Without TG2, alignment in the $\mathrm{X}$ direction was enhanced in the presence of flow. The fiber distribution ratio for each sector of the sphere confirmed that $13.7 \%$ of the fibers were aligned with $\mathrm{X}$ (axis 1) in case 2 compared to $11.8 \%$ in case 1 (see Figure 3C). Such difference apparently comes from a reduction in the ratio of fibers aligned in $Z$ (17.5\% compared to $12.1 \%$ ) and other directions with the $Z$ component (axes 5 to 12 ).

In the presence of TG2, our results show that collagen network alignment was mainly unaffected (case 4, right bottom panel, compared to case 3, left bottom panel, in Figure 3B). With such crosslinking, the ratio of fibers remained practically constant for all grouping directions before and after flow.
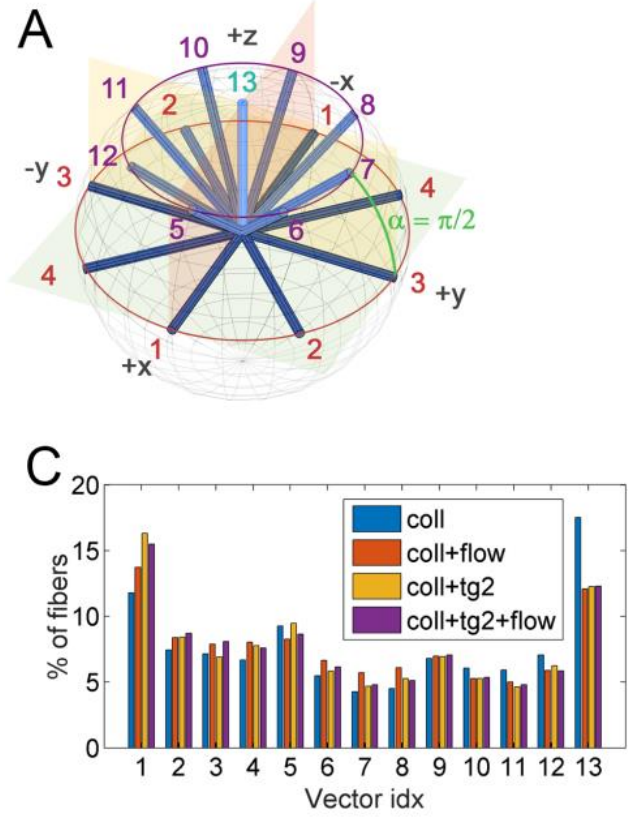
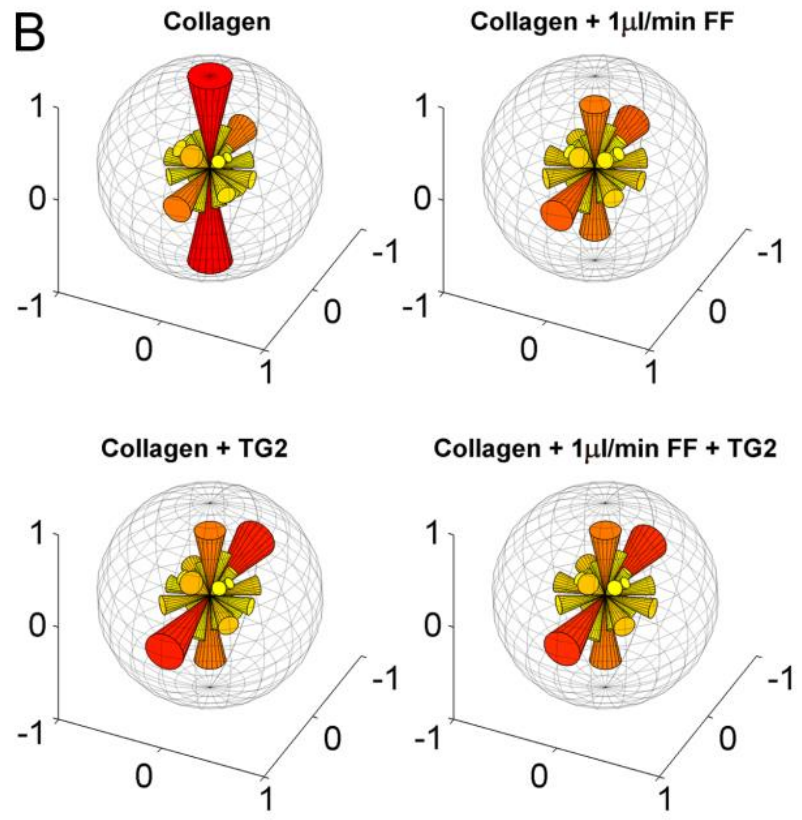

This is the post-print version of the following article: Del Amo, C., Olivares, V., Cóndor, M., Blanco, A., Santolaria, J., Asín, J., Borau C \& García-Aznar, J. M. (2018). Matrix architecture plays a pivotal role in 3D osteoblast migration: The effect of interstitial fluid flow. Journal of the mechanical behavior of biomedical materials, 83, 52-62. doi: 10.1016/j.jmbbm.2018.04.007, which has been published in final form here. 
Figure 3. A) Reference semi-sphere with numeration of the divisions used to plot the spatial fiber orientation. B) 3D rose diagrams of fiber orientation for the different conditions. C) Percentage of fibers in the corresponding sector for each condition.

\subsection{Porosity and pore size are unaffected by fluid flow}

From the reconstruction of cross-sectional images obtained by confocal microscopy, the porosity and pore size between fibers were quantified (see table 2). The distribution of pore size is obtained by fitting the Rayleigh distribution for every assay, and then, the mean value and quantiles are inferred from the model. The Rayleigh distribution is asymmetrical, showing greater differences in higher quantiles. For instance, the 90th quantile of the radius (i.e., the value that is only exceeded by $10 \%$ of the values) for TG 2 treatment groups was $2.9 \mu \mathrm{m}$ compared to the control, where $20 \%$ of the pores had a radius greater than this value (table S3 shows the data results for other quantiles). Despite the fact that the average of the mean values of pore size in TG2 treatment groups was similar for both conditions, the addition of this crosslinker induced greater homogeneity of the pore size.

Statistical analysis of three independent and different treatments for each condition showed no significant differences between them. However, the analysis of the standard deviation of the mean (Mean SD) of each condition did reveal interesting differences between them. In fact, pore size was made uniform by adding a crosslinker to the gel, especially in the case of collagen with flow application (Mean SD 0.036). The application of interstitial fluid flow resulted in a reduction of the mean standard deviation value by half in both conditions (collagen and collagen with TG2). Therefore, this data suggests that flow tends to homogenize the pore size distribution of collagen networks.

\begin{tabular}{|c|c|c|c|c|c|c|}
\cline { 2 - 7 } \multicolumn{1}{c|}{} & \multicolumn{4}{c|}{ Pore size $(\mu \mathrm{m})$} & \multicolumn{2}{c|}{ Porosity } \\
\cline { 2 - 7 } \multicolumn{1}{c|}{ Collagen } & Mean & Mean SD & Q90 & P(radius $>2.9)$ & Mean & Mean SD \\
\hline Collagen + 1 $\boldsymbol{\mu l} /$ min FF & 2 & 0.386 & 3.43 & 0.20 & 90.787 & 3.237 \\
\hline Collagen + TG2 & 1.743 & 0.137 & 2.99 & 0.12 & 91.147 & 3.58 \\
\hline Collagen + 1 $\boldsymbol{\mu l} / \mathbf{m i n}$ FF+ TG2 & 1.69 & 0.079 & 2.90 & 0.10 & 86.567 & 2.832 \\
\hline
\end{tabular}

Table 2. Values of porosity and pore size quantified from the matrix architecture and fiber distribution analyses.

\subsection{Fluid flow enhances long-distance migration}

Effective and mean velocities were obtained for HOB cells (Figure 4A). Due to the large amount of data processed in this analysis, most of the cases revealed significant differences compared to control conditions (case 1). Actually, these differences were not very relevant except for non-crosslinked collagen gels under $1 \mu \mathrm{l} /$ min flow application, which showed mean speeds with a median value approximately $40 \%$ higher and effective speeds more than twice as large as control speeds. Table 3 summarizes the obtained data.

\begin{tabular}{|c|c|c|c|c|}
\cline { 2 - 5 } & \multicolumn{2}{|c|}{ Vmean $(\mu \mathrm{m} / \mathrm{min})$} & \multicolumn{2}{c|}{ Veffective $(\mu \mathrm{m} / \mathrm{min})$} \\
\cline { 2 - 5 } & Mean \pm SD & Median & Mean \pm SD & Median \\
\hline
\end{tabular}

This is the post-print version of the following article: Del Amo, C., Olivares, V., Cóndor, M., Blanco, A., Santolaria, J., Asín, J., Borau C \& García-Aznar, J. M. (2018). Matrix architecture plays a pivotal role in 3D osteoblast migration: The effect of interstitial fluid flow. Journal of the mechanical behavior of biomedical materials, 83, 52-62. doi: 10.1016/j.jmbbm.2018.04.007, which has been published in final form here. 


\begin{tabular}{|c|c|c|c|c|}
\hline Collagen & $\begin{array}{c}0.0953 \\
\pm 0.1143\end{array}$ & 0.0556 & $\begin{array}{c}0.0153 \\
\pm 0.0152\end{array}$ & 0.0113 \\
\hline Collagen + TG2 & $\begin{array}{c}0.0990 \\
\pm 0.1208 * *\end{array}$ & 0.0560 & $\begin{array}{c}0.0223 \\
\pm 0.0212 * * *\end{array}$ & 0.0178 \\
\hline Collagen $+1 \mu \mathrm{l} / \mathrm{min} \mathrm{FF}$ & $\begin{array}{c}0.1276 \\
\pm 0.1492 * * *\end{array}$ & 0.0792 & $\begin{array}{c}0.0358 \\
\pm 0.0447 * * *\end{array}$ & 0.0216 \\
\hline $\begin{array}{c}\text { Collagen }+1 \mu \mathrm{l} / \mathrm{min} \mathrm{FF}+ \\
\text { TG2 }\end{array}$ & $\begin{array}{r}0.0899 \\
\pm 0.1051\end{array}$ & 0.0533 & $\begin{array}{c}0.0111 \\
\pm 0.0093 * * *\end{array}$ & 0.0156 \\
\hline Collagen $+0.1 \mu \mathrm{l} / \mathrm{min} \mathrm{FF}$ & $\begin{array}{c}0.0979 \\
\pm 0.1222 * * *\end{array}$ & 0.0537 & $\begin{array}{c}0.0220 \\
\pm 0.0218 * * *\end{array}$ & 0.0082 \\
\hline
\end{tabular}

Table 3. Values obtained in the cell tracking measurements of the mean and effective velocity. Each condition comprises 3 repetitions ( $\sim 60$ cells analyzed per repetition). Anova tests were performed for statistical significance. $* * * \mathrm{p}<0.001 ; * * \mathrm{p}<0.01 ; * \mathrm{p}<0.05$.

These differences are also noticeable in the relative trajectories (Figure 5), where case 2 (collagen +1 $\mu 1 / \mathrm{min}$ FF) stands out with larger and more directed paths. However, the evolution of the directionality ratio (the minimum distance between two points divided by the real covered distance; Figure 4B) showed few differences overall, indicating that $\mathrm{HOB}$ cells in these conditions tend to remain around small areas and are predominated by short and random movements.

Diffusion coefficients were also calculated for each condition and indicate the purely diffusive movement (Brownian motion) of cells in the presence of only flow and without TG2 (see table 4). However, in the rest of the cases, all migratory patterns corresponded to subdiffusive movement, which normally occurs under 3D migration conditions [36,46]. It is worth noting that both collagen $+\mathrm{TG} 2$ and collagen $+1 \mu \mathrm{m} / \mathrm{ml}$ + FF confidence intervals of the diffusivity coefficient overlap with the one from collagen, however, it is also clear that all condition mean $D$ values are out of the confidence interval of the control case (collagen) $[0.06876-0.07768]$, specially case 3 (collagen $+1 \mu \mathrm{l} / \mathrm{min} \mathrm{FF}$ ), which suggests that there are robust differences between the tested conditions and the control.

\begin{tabular}{|c|c|c|c|}
\cline { 2 - 4 } \multicolumn{1}{c|}{} & $\begin{array}{c}\text { Diffusivity } \\
\text { coefficient } \\
(\mu \mathrm{m} / \mathrm{min})(\mathrm{mean} \\
\pm \mathrm{CI})\end{array}$ & $\begin{array}{c}\text { Alpha } \\
(\mathrm{mean} \pm \mathrm{SD})\end{array}$ & Cell movement \\
\hline Collagen & $0.07322 \pm 0.00446$ & $0.83 \pm 0.28$ & Subdiffusive*** \\
\hline Collagen + TG2 & $0.07884 \pm 0.00394$ & $0.87 \pm 0.25$ & Subdiffusive*** \\
\hline $\begin{array}{c}\text { Collagen }+ \\
\mathbf{1} \boldsymbol{\mu l} / \mathbf{m i n} \text { FF }\end{array}$ & $0.2652 \pm 0.0107$ & $0.97 \pm 0.32$ & $\begin{array}{c}\text { Purely diffusive } \\
\text { (Brownian motion })\end{array}$ \\
\hline $\begin{array}{c}\text { Collagen }+ \\
\mathbf{1} \boldsymbol{\mu l} / \mathbf{m i n} \text { FF }+ \\
\mathbf{T G 2}\end{array}$ & $0.08070 \pm 0.00531$ & $0.89 \pm 0.25$ & Subdiffusive*** \\
\hline $\begin{array}{c}\text { Collagen }+ \\
\mathbf{0 . 1} \boldsymbol{\mu l} / \mathbf{m i n} \text { FF }\end{array}$ & $0.04419 \pm 0.00346$ & $0.73 \pm 0.24$ & Subdiffusive*** \\
\hline
\end{tabular}

This is the post-print version of the following article: Del Amo, C., Olivares, V., Cóndor, M., Blanco, A., Santolaria, J., Asín, J., Borau C \& García-Aznar, J. M. (2018). Matrix architecture plays a pivotal role in 3D osteoblast migration: The effect of interstitial fluid flow. Journal of the mechanical behavior of biomedical materials, 83, 52-62. doi: 10.1016/j.jmbbm.2018.04.007, which has been published in final form here. 
Table 4. Diffusivity coefficient of each condition and exponential adjustment parameter (alpha) obtained from the minimum square displacement (MSD) curve fitting [35,56]. Each condition comprises 3 repetitions ( $\sim 60$ cells analyzed per repetition). T-tests were performed against the null hypothesis that $\alpha=1$ (purely diffusive motion) $* * * \mathrm{p}<0.001$; $* * \mathrm{p}<0.01 ; * \mathrm{p}<0.05$.
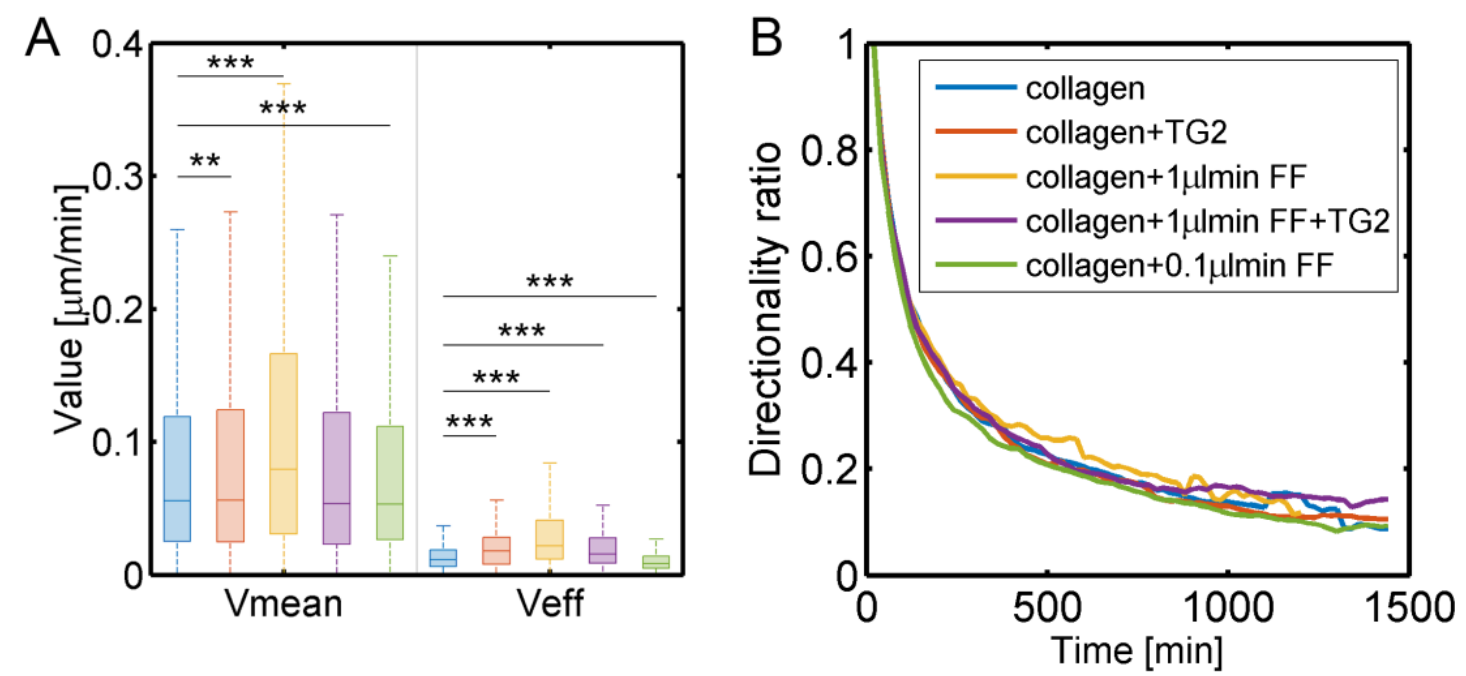

Figure 4. A) Mean and effective speed of HOB cells. Each condition comprises 3 repetitions ( $\sim 60$ cells analyzed per repetition). Anova tests were performed for statistical significance. $* * * p<0.001 ; * * p<0.01 ; * p<0.05$. B) Directionality ratio over time for each condition.

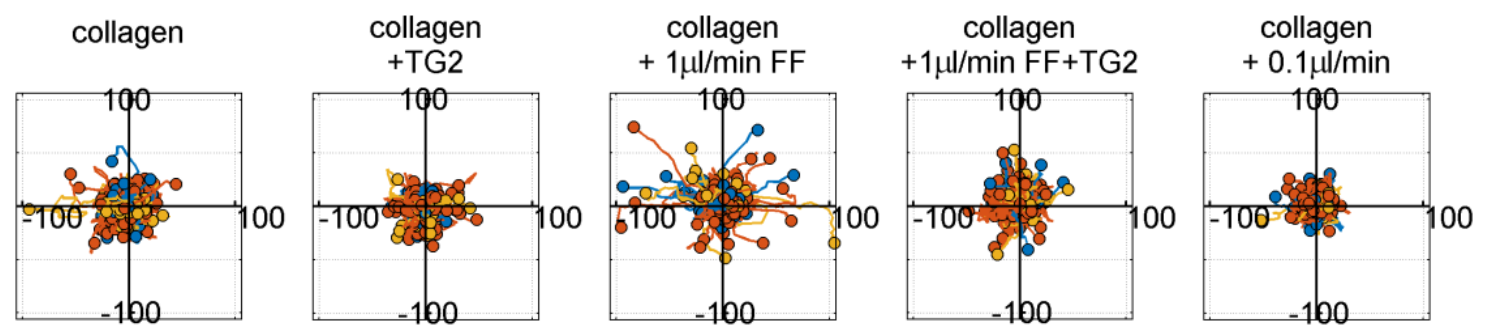

Figure 5. Trajectories of cells whose starting point was centered on the coordinate origin. The conditions analyzed were collagen or collagen + TG2 in the presence or absence of fluid flow, and the cells embedded in the matrix were altered with $1 \mu \mathrm{l} / \mathrm{min}$ of fluid flow, presenting higher directionality.

\subsection{Cell morphology is regulated by the fluid flow and the matrix architecture}

Flow was able not only to change fiber disposition but also to affect cell shape. For instance, apparently, more focal adhesions (h-Vin1 dyed with AlexaFluor ${ }^{\circledR} 488$ ) were observed in cells subjected to a $1 \mu 1 / \mathrm{min}$ flow rate compared to controls (see Figure 6A, second and fourth panel). This difference is more remarkable for cells embedded in collagen gels without TG2 crosslinker addition, presenting greater alteration in the distribution of fibers under flow application. However, a deeper analysis including a proper quantification

This is the post-print version of the following article: Del Amo, C., Olivares, V., Cóndor, M., Blanco, A., Santolaria, J., Asín, J., Borau C \& García-Aznar, J. M. (2018). Matrix architecture plays a pivotal role in 3D osteoblast migration: The effect of interstitial fluid flow. Journal of the mechanical behavior of biomedical materials, 83, 52-62. doi: 10.1016/j.jmbbm.2018.04.007, which has been published in final form here. 
of this data should be performed to confirm such observations. In any case, it is clear that TG2 increases the strength and deformation resistance of collagen hydrogels, thus decreasing fiber alterations when the gel is subjected to shear stress and in turn causing the cell morphology to change less abruptly. The formation of thin membranes between fibers confers these properties to the hydrogel without varying its stiffness significantly, as was concluded in a previous work [35]. In hydrogels with TG2, the cells showed a lobopodial shape probably as an adaptation to the matrix architecture (see Figure 6).
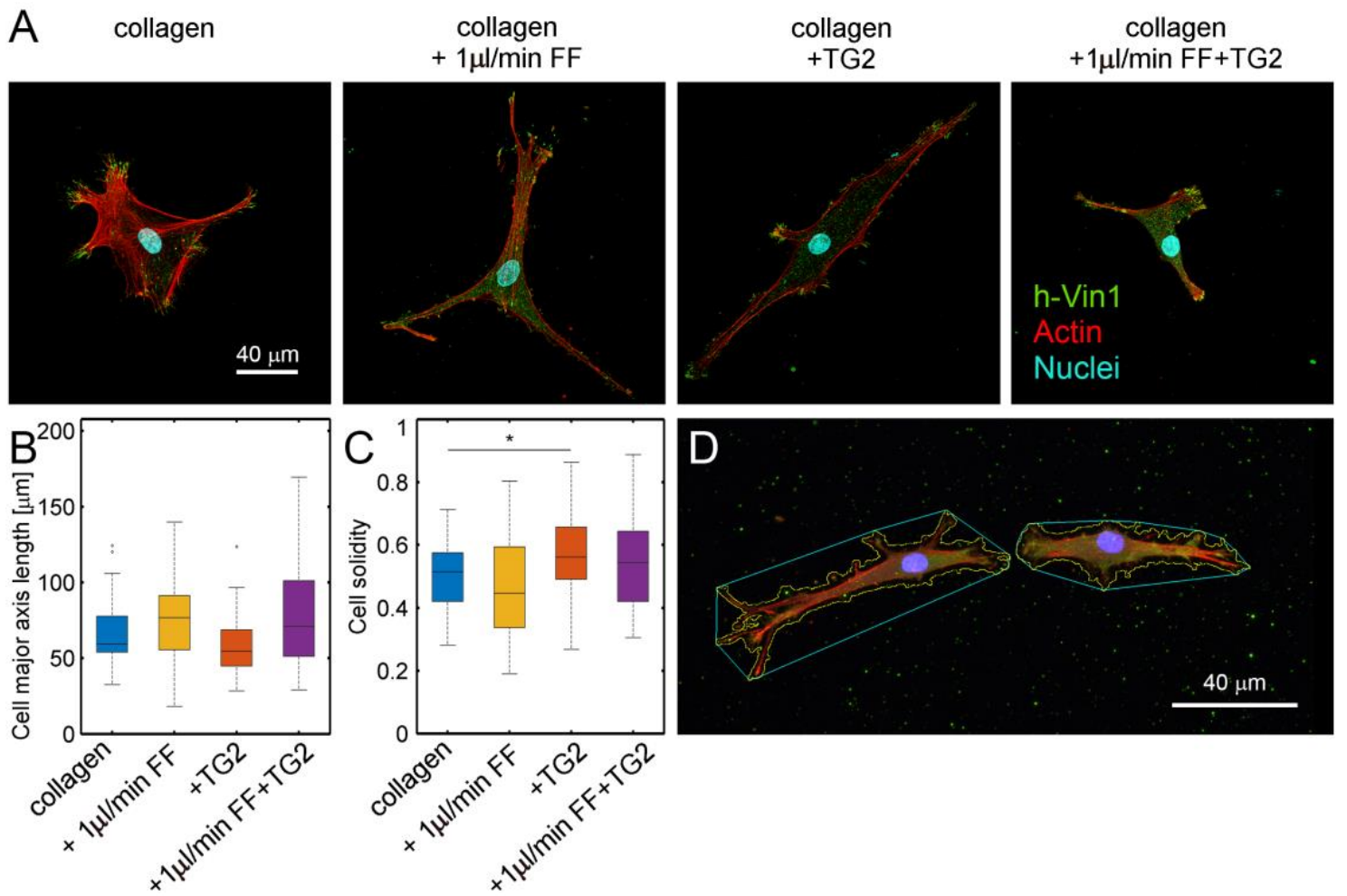

Figure 6. A) Examples of cell morphologies in the different mechanical conditions. Samples were fixed; stained for vinculin (green), phalloidin (actin, red) and DAPI (nuclei, blue); and imaged using an Olympus Fluoview FV10i confocal microscope with a $60 \mathrm{XW}$ objective. B) Cell major axis length. C) Cell solidity. D) An example of a cell processed with the MATLAB script. Segmented perimeter is highlighted in yellow. Convex polygons used for the solidity calculation are shown in cyan. Image captured using a Nikon D-Eclipse C1 confocal microscope with a 40X oil objective. $\mathrm{N} \geq 20$ cells analyzed for each condition. Anova tests were performed for statistical significance. $* * * \mathrm{p}<0.001 ; * * \mathrm{p}<0.01 ; * \mathrm{p}<0.05$.

According to the quantitative study of cell morphology from immunostained samples, cells of hydrogels crosslinked with TG2 showed higher cell solidity values compared to those without TG2. For instance, the median of the hydrogel with TG2 $(0.5613 \pm 0.1484)$ was over the $75^{\text {th }}$ quantile of cell solidity in the collagen assay (Figure 6C, first column). Even with flow, the median values obtained (TG2+flow: $0.543 \pm 0.156$ ) were greater than those corresponding to cases 1 and 2 (collagen: 0.515 \pm 0.1086 ; collagen +flow: $0.4469 \pm 0.1563)$. Thus, the data indicated that a relation between the fixation of fibers through crosslinkers and cell morphology may exist, with a lower number of protrusions and greater elongation presented in these cases compared to the control condition. In sum, cell solidity was decreased by fluid flow application, more remarkably in hydrogels without TG2.

This is the post-print version of the following article: Del Amo, C., Olivares, V., Cóndor, M., Blanco, A., Santolaria, J., Asín, J., Borau C \& García-Aznar, J. M. (2018). Matrix architecture plays a pivotal role in 3D osteoblast migration: The effect of interstitial fluid flow. Journal of the mechanical behavior of biomedical materials, 83, 52-62. doi: 10.1016/j.jmbbm.2018.04.007, which has been published in final form here. 
Thus, the application of flow decreases cell solidity and increases cell major axis length for both crosslinked and non-crosslinked gels. That is, fluid flow affects cell morphology, promoting thinner bodies and starlike shapes (Figure 6B). On the other hand, TG2 increases solidity and decreases cell major axis length overall probably due to the higher stability of the network. Figure 6D shows an example of cells segmented and processed with the hand-coded MATLAB script.

\section{Discussion}

The wide variety of mechanisms involved in cell migration are difficult to study under in vivo conditions due to the large number of factors involved in this process, from adjacent cells and tissues to intra- and extracellular signaling. This fact has promoted the development of new techniques that allow the re-creation of in vivo conditions in simplified approaches. Microfluidics is a fundamental technique for 3D culture, providing 4D monitoring (space + time), easy application of interstitial fluid flow, possibility of multiple co-culture or high control of mechano-chemical gradients among other advantages. Despite the potential of 3D cultures to mimic in vivo conditions better than traditional 2D cultures, they are still a crude approach to reproduce the physiological conditions of bone fracture healing processes. One of the main limitations is the recreation of the exact extracellular matrix surrounding bone fractures. Leong and Morgan analyzed the material properties of individual callus tissues by nanoindentation obtaining the following values of indentation moduli: granulation tissue: $0.61-1.27 \mathrm{MPa}$ (median =0.99 MPa); chondroid tissue and 1.39$4.42 \mathrm{MPa}$ (median $=2.89 \mathrm{MPa}$ ) and woven bone $26.92-1010.00 \mathrm{MPa}$ (median $=132.00 \mathrm{MPa}$ ) [57]. Compared to those values, the material properties of our collagen-based gels are low (see Table 1). Although we could tune these properties using different types of gels, it is very difficult to find natural or synthetic ones whose stiffness and architecture get close to those of granulation tissue, soft callus or hard callus. Another limitation of the microfluidic approach to simulate fracture healing is that normally the interfragmentary gap suffers some strains that induce the movement of interstitial fluid flow. This effect is actually not included in this in-vitro analysis, because we can only simulate the interstitial fluid conditions directly. Although we have mainly applied unidirectional fluid flow, oscillatory fluid flow would be more representative of fracture healing conditions and will be studied in future works. Even with its limitations, our microfluidic system allows us to analyze the isolated and combined effect of different microenvironmental stimuli on osteoblast behavior. This permits to advance in the understanding of how interstitial fluid flow and the hardening of the matrix (by means of crosslinking) may affect the osteoblast navigation capacity in $3 \mathrm{D}$, which is the main aim of this work.

The dynamic load on the bone is fundamental for osteogenesis and maintenance of bone homeostasis, regulating the different cellular responses. The mechanosensitivity response of osteoblasts has been previously studied mainly in cells from murine species; for example, the molecular aspects involved in ion transport, such as calcium (receptors (P2Y2)) [58] or nitric oxide release in osteoblasts, have been analyzed [59]. Additionally, the potent stimulator effect of fluid flow on ECM synthesis has been reported by other authors [60-62], some of them concluding that interstitial fluid flow enhances the creation of bone matrix after a few days [63].

Physiologically, during the formation of bone tissue, osteoblasts synthesize and deposit type I collagen, either with a random orientation of collagen fibrils (woven bone) or grouped in parallel matrices (lamellar bone) following an orthogonal pattern [64]. Hoert et al. have proposed a model (based on murine bone) where they analyzed the nanostructure and micromechanical properties of the new corpus callosum and mature laminar bone in the cortex, demonstrating its different fibrillary organization and mineral content in the different types of healing bone $[65,66]$. In an attempt to recreate this collagen-based matrix in the woven bone, in our experiments the disposition of collagen fibers was modified by altering the collagen

This is the post-print version of the following article: Del Amo, C., Olivares, V., Cóndor, M., Blanco, A., Santolaria, J., Asín, J., Borau C \& García-Aznar, J. M. (2018). Matrix architecture plays a pivotal role in 3D osteoblast migration: The effect of interstitial fluid flow. Journal of the mechanical behavior of biomedical materials, 83, 52-62. doi: 10.1016/j.jmbbm.2018.04.007, which has been published in final form here. 
concentration (see S5- Supplementary Material) as well as by the addition of TG2. In this context, the study of fluid flow effects on HOB migration in terms of mean (instantaneous) and effective velocities revealed significant differences, especially when a $1 \mu \mathrm{l} / \mathrm{ml}$ fluid flow rate was applied to gels without TG2. Matrix structural analysis revealed that the fiber distribution in crosslinked gels remained unaltered despite the application of flow, while significant alignment with flow occurred in non-crosslinked gels. We consider that this effect may determine the different migratory pattern. It is worth mentioning that age usually increases the concentration of collagen crosslinkers, inducing the stiffening of the ECM [67], which sometimes can be associated to diabetes [68]. In these situations, one of the main biochemical abnormalities that occur is the formation of advanced glycation end-products (AGEs) as a consequence of non-enzymatic collagen glycosylation, directly related to increased collagen crosslinking [69]. Therefore, our in-vitro model allows simulating the process of aging by means of increasing the concentration of collagen crosslinkers, showing a lower capacity in the osteoblast migration in comparison with crosslinker-free collagen gels. This effect is in agreement with the delay healing that occurs in diabetic and elder patients.

Nevertheless, other effects might be also regulating osteoblast motility, for example, fluid flow might directly affect cells and alteration of fibers might modify mechanical and degradation properties of these collagen-based gels. In fact, osteoblast migration in 3D is mainly determined by the capacity of osteoblasts to deform and degrade the matrix [35]. In a similar way, when analyzing non-crosslinked gels, we observe that high rates of flow enhanced osteoblast velocity, but these rates also produced changes in cell morphology, with the cells acquiring a more star-like appearance. Finally, when studying migration on crosslinked gels, we found that osteoblast migration remained mostly unaffected despite fluid flow, while their morphology changed similar to those in non-crosslinked gels. Hence, these results also suggest that other stimuli like direct fluid flow sensed by cells might be regulating osteoblast morphology. In addition, we can observe that the most important differences in cell morphology were observed when collagen gel was crosslinked, probably caused by the increment of the mechanical [70-72] and adhesion properties $[43,73]$ induced by the inclusion of TG2.

To understand these results, a quantitative analysis of the microstructure of gels for all conditions was performed. This quantification revealed that the addition of one crosslinker to the collagen hydrogel prevented the alteration of fiber disposition when subjected to flow. A matrix architecture analysis was performed, and the pore size and porosity were estimated, revealing a more uniform distribution of the architecture when TG2 was added. While crosslinker-free collagen gels presented very large or small pores, the application of fluid flow resulted in greater uniformity of the pore size, which may be directly affecting cell migration. In the case of gels with TG2, although the average pore size did not vary in the case without flow, the pores were less uniform than when an interstitial flow was applied, thus favoring cell movement. The differences found were, however, not significant, so more gels should be analyzed to confirm strength this observation. The addition of this stabilizer intended to simulate the different matrices to which osteoblasts are subjected during endochondral ossification. Cell migration under TG2 conditions presented patterns similar to those of the control, regardless of the presence of flow. However, the interstitial fluid flow through the matrix was able to alter cell morphology without altering the architecture of the hydrogel, which produced cells with low branching (high solidity).

Other studies of the interstitial flow effect on matrix disposition have been performed. For instance, Guo \& Kaufman [74] analyzed the effect of collagen fiber alignment on cell spreading and concluded that after several hours of alignment, this effect is reduced as the cell concentration is higher and with the consequent remodeling of the matrix and fiber aggregation. Hydrogels used in most of the published works presented a lower collagen concentration $(2 \mathrm{mg} / \mathrm{ml}, 2.5 \mathrm{mg} / \mathrm{ml})[74,75]$ compared to the collagen concentration used in this work $(4 \mathrm{mg} / \mathrm{ml})$. These differences in concentration and in the rigidity of the hydrogels can be crucial for observing a clear alignment in low-concentration gels, which are more easily modified by external

This is the post-print version of the following article: Del Amo, C., Olivares, V., Cóndor, M., Blanco, A., Santolaria, J., Asín, J., Borau C \& García-Aznar, J. M. (2018). Matrix architecture plays a pivotal role in 3D osteoblast migration: The effect of interstitial fluid flow. Journal of the mechanical behavior of biomedical materials, 83, 52-62. doi: 10.1016/j.jmbbm.2018.04.007, which has been published in final form here. 
stimuli. For the gels used in this work, it was necessary to obtain and quantify confocal microscopic images since at first sight, the differences were not perceptible.

To better understand the three-dimensional migratory pattern of osteoblasts, apart from quantifying the trajectories followed in the assays monitored over time, cells were immunostained to analyze their morphology. With our results, we could conclude that the laxity of unmodified collagen fibers allows cells to have a larger cell body, while greater polarization was found in cells embedded in hydrogels with TG2. Overall, fluid flow promoted the elongation of cells.

\section{Conclusions}

Between all the cases analyzed, we quantified significant differences in the osteoblast migration speed when collagen-based gels were exposed to interstitial fluid flow. In fact, we found that interstitial fluid flow application promotes matrix fiber alignment and homogenization of the pore size. To strengthen the collagen network we crosslinked collagen-based gels with TG2. Under these conditions, we observed no changes in osteoblast migration speed. These results together demonstrate the crucial influence of matrix architecture on osteoblast migration.

Furthermore, we observed an alteration in osteoblast morphology when interstitial flow was applied in different collagen-based gels (crosslinked or non-crosslinked). This finding suggests that interstitial flow may help to modulate osteoblast phenotypes and drive the progression of bone regeneration. Taken together, these results have a potential use in regenerative medicine and may be useful in the design of therapeutic approaches to promote bone regeneration and prevent unsuccessful bone healing.

\section{Acknowledgments}

In terms of funding this work was supported by the European Research Council (ERC) through project INSILICO-CELL (ERC-2012-StG 306571) and its proof of concept IMAGO (ERC-PoC-2016-737543); the Spanish Ministry of Economy and Competitiveness through project DPI2015-64221-C2-1-R; the Spanish Ministry of Education, Culture and Sport (FPU13/03194); and the Government of Aragon (C126/2015 MC). The authors would like to thank Professor Roger Kamm for technical assistance with the development of the microfluidic techniques, Dr. Ioannis K. Zervantonakis and Dr. William Polacheck for support with fluid flow quantification and setup, Professor Ben Fabry for his valuable help with the imagebased characterization of the hydrogel architecture, the Precision Mechanics Service of the University of Zaragoza for fabrication of the metal platforms and the Microscopy Service of CIBA (IACS- University of Zaragoza).

\section{Author Contributions}

Conceived and designed the experiments: JMGA, CDA. Performed the experiments: CDA. Analyzed the data: CDA, VO. Analysis tools: CB, VO, MC. Design of fluid flow support: CDA, AB, JS. Statistical analysis: CB, JA. Wrote the paper: CDA, JMGA, CB.

This is the post-print version of the following article: Del Amo, C., Olivares, V., Cóndor, M., Blanco, A., Santolaria, J., Asín, J., Borau C \& García-Aznar, J. M. (2018). Matrix architecture plays a pivotal role in 3D osteoblast migration: The effect of interstitial fluid flow. Journal of the mechanical behavior of biomedical materials, 83, 52-62. doi: 10.1016/j.jmbbm.2018.04.007, which has been published in final form here. 


\section{Bibliography}

[1] P. V Giannoudis, T.A. Einhorn, D. Marsh, Fracture healing: the diamond concept, Injury. (2007) 3-6.

[2] T.A. Einhorn, L.C. Gerstenfeld, Fracture healing: mechanisms and interventions, Nat Rev Rheumatol. 11 (2015) 45-54. doi:10.1038/nrrheum.2014.164.

[3] E.F. Morgan, R.E.G. Kristy T. Salisbury Palomares, D.L. Bellin, K.B. Chien, G.U. Unnikrishnan, P.L. Leong, Correlations between local strains and tissue phenotypes in an experimental model of skeletal healing, J. Biomech. 43 (2010) 2418-2424. doi:10.1016/J.JBIOMECH.2010.04.019.

[4] P.J. Prendergast, R. Huiskes, K. Soballes, Biophysical stimuli on cells during tissue differentiation at implant interfaces, J. Biomech. 30 (1997) 539-548. doi:10.1016/S0021-9290(96)00140-6.

[5] A. Thiel, M.K. Reumann, A. Boskey, J. Wischmann, R. von Eisenhart-Rothe, P. Mayer-Kuckuk, Osteoblast migration in vertebrate bone, Biol. Rev. (2017). doi:10.1111/brv.12345.

[6] A. Bailón-Plaza, M.C.H. Van Der Meulen, A Mathematical Framework to Study the Effects of Growth Factor Influences on Fracture Healing, J. Theor. Biol. 212 (2001) 191-209. doi:10.1006/jtbi.2001.2372.

[7] D. Lacroix, P.J. Prendergast, G. Li, D. Marsh, Biomechanical model to simulate tissue differentiation and bone regeneration: application to fracture healing., Med. Biol. Eng. Comput. 40 (2002) 14-21. doi:10.1007/BF02347690.

[8] M. Doblaré, J.M. García, M.J. Gómez, Modelling bone tissue fracture and healing: A review, Eng. Fract. Mech. 71 (2004) 1809-1840. doi:10.1016/j.engfracmech.2003.08.003.

[9] S.J. Shefelbine, P. Augat, L. Claes, U. Simon, Trabecular bone fracture healing simulation with finite element analysis and fuzzy logic, J. Biomech. 38 (2005) 2440-2450. doi:10.1016/j.jbiomech.2004.10.019.

[10] L. Claes, C.A.C.A. Heigele, C. Neidlinger-Wilke, D. Kaspar, W. Seidl, K.J.K.J. Margevicius, P. Augat, Effects of Mechanical Factors on the Fracture Healing Process, Clin. Orthop. Relat. Res. 355S (1998) S132S147. doi:10.1097/00003086-199810001-00015.

[11] R. Marsell, T.A. Einhorn, The biology of fracture healing, Injury. 42 (2012) 551-555. doi:10.1016/j.injury.2011.03.031.

[12] D. Holmes, Non-union bone fracture: A quicker fix, Nat. Outl. 550 (2017) S193. doi:10.1038/550S193a.

[13] A. Thiel, M.K. Reumann, A. Boskey, J. Wischmann, R. von Eisenhart-Rothe, P. Mayer-Kuckuk, Osteoblast migration in vertebrate bone, Biol. Rev. 49 (2017). doi:10.1111/brv.12345.

[14] M.J. McGarrigle, C.A. Mullen, M.G. Haugh, M.C. Voisin, L.M. McNamara, Osteocyte differentiation and the formation of an interconnected cellular network in vitro, Eur. Cells Mater. 31 (2016) 323-340. doi:10.22203/eCM.v031a21.

[15] M. Wang, N. Yang, X. Wang, A review of computational models of bone fracture healing, Med. Biol. Eng. Comput. 55 (2017) 1895-1914. doi:10.1007/s11517-017-1701-3.

[16] M.S. Ghiasi, J. Chen, A. Vaziri, E.K. Rodriguez, A. Nazarian, Bone fracture healing in mechanobiological modeling: A review of principles and methods, Bone Reports. 6 (2017) 87-100. doi:10.1016/j.bonr.2017.03.002.

[17] L.A. González-Torres, M.J. Gómez-Benito, M. Doblaré, J.M. García-Aznar, Influence of the frequency of the external mechanical stimulus on bone healing: a computational study, Med. Eng. Phys. 34 (2010) 363371.

[18] M.J. Gómez-Benito, L.A. González-Torres, E. Reina-Romo, J. Grasa, B. Seral, J.M. García-Aznar, Influence of high-frequency cyclical stimulation on the bone fracture-healing process: mathematical and experimental models, Phil. Trans. R. Soc. A. 369 (2011) 4278-4294.

[19] J.M. Garcia-Aznar, J.H. Kuiper, M.J. Gómez-Benito, M. Doblaré, J.B. Richardson, Computational

This is the post-print version of the following article: Del Amo, C., Olivares, V., Cóndor, M., Blanco, A., Santolaria, J., Asín, J., Borau C \& García-Aznar, J. M. (2018). Matrix architecture plays a pivotal role in 3D osteoblast migration: The effect of interstitial fluid flow. Journal of the mechanical behavior of biomedical materials, 83, 52-62. doi: 10.1016/j.jmbbm.2018.04.007, which has been published in final form here. 
simulation of fracture healing: influence of interfragmentary movement on the callus growth, J. Biomech. 40 (2007) 1467-1476.

[20] M.J. Gomez-Benito, J.M. Garcia-Aznar, J.H. Kuiper, M. Doblaré, Influence of fracture gap size on the pattern of long bone healing: a computational study. Journal of theoretical biology, J. Theor. Biol. 235 (2005) 105-119.

[21] H. Isaksson, W. Wilson, C.C. van Donkelaar, R. Huiskes, K. Ito, Comparison of biophysical stimuli for mechano-regulation of tissue differentiation during fracture healing, J. Biomech. 39 (2006) 1507-1516.

[22] W. Yu, H. Qu, G. Hu, Q. Zhang, K. Song, H. Guan, T. Liu, J. Qin, A Microfluidic-Based Multi-Shear Device for Investigating the Effects of Low Fluid-Induced Stresses on Osteoblasts, PLoS One. 9 (2014) e89966. doi:10.1371/journal.pone.0089966.

[23] B.D. Riehl, J.S. Lee, L. Ha, I.K. Kwon, J.Y. Lim, Flowtaxis of osteoblast migration under fluid shear and the effect of RhoA kinase silencing, PLoS One. 12 (2017) 1-16. doi:10.1371/journal.pone.0171857.

[24] M. Mullender, A.J. El Haj, Y. Yang, M.A. van Duin, E.H. Burger, J. Klein-Nulend, Mechanotransduction of bone cells in vitro: Mechanobiology of bone tissue, Med. Biol. Eng. Comput. 42 (2004) 14-21. doi:10.1007/BF02351006

[25] S.D. Tan, A.D. Bakker, C.M. Semeins, A.M. Kuijpers-Jagtman, J. Klein-Nulend, Inhibition of osteocyte apoptosis by fluid flow is mediated by nitric oxide, Biochem. Biophys. Res. Commun. 369 (2008) 1150 1154. doi:10.1016/j.bbrc.2008.03.007.

[26] N.E. Ajubi, J. Klein-Nulend, P.J. Nijweide, T. Vrijheid-Lammers, M.J. Alblas, E.H. Burger, Pulsating fluid flow increases prostaglandin production by cultured chicken osteocytes-A cytoskeleton-dependent process, Biochem. Biophys. Res. Commun. 225 (1996) 62-68. doi:10.1006/bbrc.1996.1131.

[27] I. Westbroek, N.E. Ajubi, M.J. Alblas, C.M. Semeins, J. Klein-Nulend, E.H. Burger, P.J. Nijweide, Differential stimulation of prostaglandin $\mathrm{G} / \mathrm{H}$ synthase-2 in osteocytes and other osteogenic cells by pulsating fluid flow, Biochem. Biophys. Res. Commun. 268 (2000) 414-419. doi:10.1006/bbrc.2000.2154

[28] M. V Hillsley, J. a Frangos, Bone tissue engineering: the role of interstitial fluid flow., Biotechnol. Bioeng. 43 (1994) 573-81. doi:10.1002/bit.260430706.

[29] B. Clarke, Normal bone anatomy and physiology., Clin. J. Am. Soc. Nephrol. 3 Suppl 3 (2008) S131-9. doi:10.2215/CJN.04151206.

[30] X. Feng, Chemical and Biochemical Basis of Cell-Bone Matrix Interaction in Health and Disease, Curr Chem Biol. 3 (2009) 189-196. doi:10.2174/187231309788166398.Chemical.

[31] P. Augat, M. Faschingbauer, K. Seide, K. Tobita, S.A. Callary, L.B. Solomon, J.H. Holstein, Biomechanical methods for the assessment of fracture repair, Injury. 45 (2014) S32-S38. doi:10.1016/j.injury.2014.04.006.

[32] P. Augat, U. Simon, A. Liedert, L. Claes, Mechanics and mechano-biology of fracture healing in normal and osteoporotic bone, Osteoporos. Int. 16 (2005) 36-43. doi:10.1007/s00198-004-1728-9.

[33] L. Claes, S. Recknagel, A. Ignatius, Fracture healing under healthy and inflammatory conditions, Nat. Rev. Rheumatol. 8 (2012) 133-143. doi:10.1038/nrrheum.2012.1.

[34] U. Saran, S.G. Piperni, S. Chatterjee, Role of angiogenesis in bone repair, Arch. Biochem. Biophys. 561 (2014) 109-117. doi:10.1016/j.abb.2014.07.006.

[35] N. Movilla, C. Borau, C. Valero, J.M. García-Aznar, Degradation of extracellular matrix regulates osteoblast migration : a microfluidic-based study, Bone. 107 (2018) 10-17.

[36] O. Moreno-Arotzena, C. Borau, N. Movilla, M. Vicente-Manzanares, J.M. García-Aznar, Fibroblast Migration in 3D is Controlled by Haptotaxis in a Non-muscle Myosin II-Dependent Manner, Ann. Biomed. Eng. (2015). doi:10.1007/s10439-015-1343-2.

[37] C. Del Amo, C. Borau, N. Movilla, J. Asín, J.M. Garcia-Aznar, Quantifying 3D chemotaxis in microfluidic-

This is the post-print version of the following article: Del Amo, C., Olivares, V., Cóndor, M., Blanco, A., Santolaria, J., Asín, J., Borau C \& García-Aznar, J. M. (2018). Matrix architecture plays a pivotal role in 3D osteoblast migration: The effect of interstitial fluid flow. Journal of the mechanical behavior of biomedical materials, 83, 52-62. doi: 10.1016/j.jmbbm.2018.04.007, which has been published in final form here. 
based chips with step gradients of collagen hydrogel concentrations, Integr. Biol. (2017) 1-27. doi:10.1039/C7IB00022G.

[38] Q. Sun, Y. Gu, W. Zhang, L. Dziopa, J. Zilberberg, W. Lee, Ex vivo 3D osteocyte network construction with primary murine bone cells, Bone Res. 3 (2015) 15026. doi:10.1038/boneres.2015.26.

[39] L. Prodanov, C.M. Semeins, J.J.W. a van Loon, J. te Riet, J. a Jansen, J. Klein-Nulend, X.F. Walboomers, Influence of nanostructural environment and fluid flow on osteoblast-like cell behavior: a model for cellmechanics studies., Acta Biomater. 9 (2013) 6653-62. doi:10.1016/j.actbio.2013.02.011.

[40] L.H. Nguyen, N. Annabi, M. Nikkhah, H. Bae, L. Binan, S. Park, Y. Kang, Y. Yang, A. Khademhosseini, Vascularized bone tissue engineering: approaches for potential improvement., Tissue Eng. Part B. Rev. 18 (2012) 363-82. doi:10.1089/ten.TEB.2012.0012.

[41] Y. Li, Y. Luo, K. Huang, J. Xing, Z. Xie, M. Lin, L. Yang, Y. Wang, The responses of osteoblasts to fluid shear stress depend on substrate chemistries, Arch. Biochem. Biophys. 539 (2013) 38-50. doi:10.1016/j.abb.2013.09.005.

[42] S.I. Fraley, P.-H. Wu, L. He, Y. Feng, R. Krisnamurthy, G.D. Longmore, D. Wirtz, Three-dimensional matrix fiber alignment modulates cell migration and MT1-MMP utility by spatially and temporally directing protrusions., Sci. Rep. 5 (2015) 14580. doi:10.1038/srep14580.

[43] H. Tatsukawa, Y. Furutani, K. Hitomi, S. Kojima, Transglutaminase 2 has opposing roles in the regulation of cellular functions as well as cell growth and death, 7 (2016) e2244-12. doi:10.1038/cddis.2016.150.

[44] M.T. Kaartinen, S. El-Maadawy, N.H. Rasanen, M.D. McKee, Tissue transglutaminase and its substrates in bone, J. Bone Miner. Res. 17 (2002) 2161-2173. doi:10.1359/jbmr.2002.17.12.2161.

[45] W.A. Farahat, L.B. Wood, I.K. Zervantonakis, A. Schor, S. Ong, D. Neal, R.D. Kamm, H.H. Asada, Ensemble analysis of angiogenic growth in three-dimensional microfluidic cell cultures., PLoS One. 7 (2012) e37333. doi:10.1371/journal.pone.0037333.

[46] C. Del Amo, C. Borau, R. Gutiérrez, J. Asín, J.M. García-Aznar, Quantification of angiogenic sprouting under different growth factors in a microfluidic platform, J. Biomech. 49 (2016) 1340-1346. doi:10.1016/j.jbiomech.2015.10.026.

[47] C.-Y. Fu, S.-Y. Tseng, S.-M. Yang, L. Hsu, C.-H. Liu, H.-Y. Chang, A microfluidic chip with a U-shaped microstructure array for multicellular spheroid formation, culturing and analysis., Biofabrication. 6 (2014) 15009. doi:10.1088/1758-5082/6/1/015009.

[48] J.P. Kruth, M.C. Leu, T. Nakagawa, Progress in additive manufacturing and rapid prototyping, CIRP Ann. Manuf. Technol. 47 (1998) 525-540. doi:10.1016/S0007-8506(07)63240-5.

[49] D. Hoshino, K.C. Kirkbride, K. Costello, E.S. Clark, S. Sinha, N. Grega-Larson, M.J. Tyska, A.M. Weaver, Exosome secretion is enhanced by invadopodia and drives invasive behavior, Cell Rep. 5 (2013). doi:10.1007/s11103-011-9767-z.Plastid.

[50] K.E. Sung, G. Su, C. Pehlke, S.M. Trier, K.W. Eliceiri, P.J. Keely, A. Friedl, D.J. Beebe, Control of 3 dimensional collagen matrix polymerization for reproducible Human Mammary Fibroblast cell culture in microfluidic devices Kyung, Biomaterials. 30 (2009) 4833-4841. doi:10.1016/j.biomaterials.2009.05.043.Control.

[51] J.S. Bredfeldt, Y. Liu, C.A. Pehlke, M.W. Conklin, J.M. Szulczewski, D.R. Inman, P.J. Keely, R.D. Nowak, T.R. Mackie, K.W. Eliceiri, Computational segmentation of collagen fibers from second-harmonic generation images of breast cancer, J. Biomed. Opt. 19 (2014) 16007. doi:10.1117/1.JBO.19.1.016007.

[52] A.M. Stein, D.A. Vader, L.M. Jawerth, D.A. Weitz, L.M. Sander, An algorithm for extracting the network geometry of three-dimensional collagen gels, J. Microsc. 232 (2008) 463-475. doi:10.1111/j.13652818.2008.02141.x.

[53] N.R. Lang, S. Münster, C. Metzner, P. Krauss, S. Schürmann, J. Lange, K.E. Aifantis, O. Friedrich, B. Fabry, Estimating the 3D pore size distribution of biopolymer networks from directionally biased data,

This is the post-print version of the following article: Del Amo, C., Olivares, V., Cóndor, M., Blanco, A., Santolaria, J., Asín, J., Borau C \& García-Aznar, J. M. (2018). Matrix architecture plays a pivotal role in 3D osteoblast migration: The effect of interstitial fluid flow. Journal of the mechanical behavior of biomedical materials, 83, 52-62. doi: 10.1016/j.jmbbm.2018.04.007, which has been published in final form here. 
Biophys. J. 105 (2013) 1967-1975. doi:10.1016/j.bpj.2013.09.038.

[54] N. Tarantino, J.-Y. Tinevez, E.F. Crowell, B. Boisson, R. Henriques, M. Mhlanga, F. Agou, A. Israël, E. Laplantine, TNF and IL-1 exhibit distinct ubiquitin requirements for inducing NEMO-IKK supramolecular structures, J Cell Biol. 201 (2014) 231-45.

[55] O. Moreno-Arotzena, J. Meier, C. del Amo, J. García-Aznar, Characterization of Fibrin and Collagen Gels for Engineering Wound Healing Models, Materials (Basel). 8 (2015) 1636-1651. doi:10.3390/ma8041636.

[56] P.-H. Wu, A. Giri, D. Wirtz, Statistical analysis of cell migration in 3D using the anisotropic persistent random walk model., Nat. Protoc. 10 (2015) 517-27. doi:10.1038/nprot.2015.030.

[57] P.L. Leong, E.F. Morgan, Measurement of fracture callus material properties via nanoindentation, Acta Biomater. 4 (2008) 1569-1575. doi:10.1016/j.actbio.2008.02.030.Measurement.

[58] J. Gardinier, W. Yang, G.R. Madden, A. Kronbergs, V. Gangadharan, E. Adams, K. Czymmek, R.L. Duncan, P2Y2 receptors regulate osteoblast mechanosensitivity during fluid flow., Am. J. Physiol. Cell Physiol. 306 (2014) C1058-67. doi:10.1152/ajpcell.00254.2013.

[59] D.L. Johnson, T.N. McAllister, J. a Frangos, Fluid flow stimulates rapid and continuous release of nitric oxide in osteoblasts., Am. J. Physiol. 271 (1996) E205-8. doi:papers://82E9EA27-E255-4A82-9E406DAC45A310F4/Paper/p499.

[60] E. Birmingham, T.C. Kreipke, E.B. Dolan, T.R. Coughlin, P. Owens, L.M. McNamara, G.L. Niebur, P.E. McHugh, Mechanical Stimulation of Bone Marrow In Situ Induces Bone Formation in Trabecular Explants, Ann. Biomed. Eng. 43 (2015) 1036-1050. doi:10.1007/s10439-014-1135-0.

[61] H.L. Morris, C.I. Reed, J.W. Haycock, G.C. Reilly, Mechanisms of fluid-flow-induced matrix production in bone tissue engineering, Proc. Inst. Mech. Eng. Part H J. Eng. Med. 224 (2010) 1509-1521. doi:10.1243/09544119JEIM751.

[62] Y.-Y. Jia, F. Li, N. Geng, P. Gong, S.-J. Huang, L.-X. Meng, J. Lan, Y. Ban, Fluid flow modulates the expression of genes involved in the Wnt signaling pathway in osteoblasts in 3D culture conditions, Int. J. Mol. Med. 33 (2014) 1282-1288. doi:10.3892/ijmm.2014.1694.

[63] E.A. Botchwey, S.R. Pollack, S. El-Amin, E.M. Levine, R.S. Tuan, C.T. Laurencin, Human osteoblast-like cells in three-dimensional culture with fluid flow, Biorheology. 40 (2003) 299-306.

[64] F. Shapiro, Bone development and its relation to fracture repair. The roleof mesenchymal osteoblasts and surface osteoblasts, Eur. Cells Mater. 15 (2008) 53-76. doi:vo1015a05 [pii].

[65] R.M. Hoerth, B.M. Seidt, M. Shah, C. Schwarz, B.M. Willie, G.N. Duda, P. Fratzl, W. Wagermaier, Mechanical and structural properties of bone in non-critical and critical healing in rat, Acta Biomater. 10 (2014) 4009-4019. doi:10.1016/j.actbio.2014.06.003.

[66] R.M. Hoerth, M. Kerschnitzki, M. Aido, I. Schmidt, M. Burghammer, G.N. Duda, P. Fratzl, B.M. Willie, W. Wagermaier, Correlations between nanostructure and micromechanical properties of healing bone, J. Mech. Behav. Biomed. Mater. 77 (2018) 258-266. doi:10.1016/j.jmbbm.2017.08.022.

[67] L.K. Wood, E. Kayupov, J.P. Gumucio, C.L. Mendias, D.R. Claflin, S. V. Brooks, Intrinsic stiffness of extracellular matrix increases with age in skeletal muscles of mice, J. Appl. Physiol. 117 (2014) 363-369. doi:10.1152/japplphysiol.00256.2014.

[68] V.M. Monnier, G.T. Mustata, K.L. Biemel, O. Reihl, M.O. Lederer, D. Zhenyu, D.R. Sell, Cross-linking of the extracellular matrix by the Maillard reaction in aging and diabetes: An update on "a puzzle nearing resolution,” Ann. N. Y. Acad. Sci. 1043 (2005) 533-544. doi:10.1196/annals.1333.061.

[69] M. Abate, C. Schiavone, P. Pelotti, V. Salini, Limited joint mobility in diabetes and ageing: recent advances in pathogenesis and therapy., Int. J. Immunopathol. Pharmacol. 23 (2010) 997-1003. doi:10.1177/039463201002300404.

[70] D. Fortunati, D.Y.S. Chau, Z. Wang, R.J. Collighan, M. Griffin, Cross-linking of collagen I by tissue

This is the post-print version of the following article: Del Amo, C., Olivares, V., Cóndor, M., Blanco, A., Santolaria, J., Asín, J., Borau C \& García-Aznar, J. M. (2018). Matrix architecture plays a pivotal role in 3D osteoblast migration: The effect of interstitial fluid flow. Journal of the mechanical behavior of biomedical materials, 83, 52-62. doi: 10.1016/j.jmbbm.2018.04.007, which has been published in final form here. 
transglutaminase provides a promising biomaterial for promoting bone healing, Amino Acids. 46 (2014) 1751-1761. doi:10.1007/s00726-014-1732-0.

[71] J.M. Orban, L.B. Wilson, J.A. Kofroth, M.S. El-Kurdi, T.M. Maul, D.A. Vorp, Crosslinking of collagen gels by transglutaminase, J. Biomed. Mater. Res. 68A (2004) 756-762. doi:10.1002/jbm.a.20110.

[72] C. Valero, H. Amaveda, M. Mora, J.M. Garcia-aznar, Combined experimental and computational characterization of crosslinked collagen- based hydrogels, PLoS One. (accepted) (2018).

[73] E. Png, L. Tong, Transglutaminase-2 in cell adhesion: All roads lead to paxillin?, Cell Adhes. Migr. 7 (2013) 412-417. doi:10.4161/cam.26344.

[74] C. Guo, L.J. Kaufman, Flow and magnetic field induced collagen alignment, Biomaterials. 28 (2007) 11051114. doi:10.1016/j.biomaterials.2006.10.010.

[75] C.P. Ng, B. Hinz, M. a Swartz, Interstitial fluid flow induces myofibroblast differentiation and collagen alignment in vitro., J. Cell Sci. 118 (2005) 4731-9. doi:10.1242/jcs.02605.

[76] J.I. Villalta, S. Galli, M.F. Iacaruso, V.G.A. Arciuch, J.J. Poderoso, E.A. Jares-Erijman, L.I. Pietrasanta, New algorithm to determine true colocalization in combination with image restoration and time-lapse confocal microscopy to map Kinases in mitochondria, PLoS One. 6 (2011) 1-16. doi:10.1371/journal.pone.0019031.

This is the post-print version of the following article: Del Amo, C., Olivares, V., Cóndor, M., Blanco, A., Santolaria, J., Asín, J., Borau C \& García-Aznar, J. M. (2018). Matrix architecture plays a pivotal role in 3D osteoblast migration: The effect of interstitial fluid flow. Journal of the mechanical behavior of biomedical materials, 83, 52-62. doi: 10.1016/j.jmbbm.2018.04.007, which has been published in final form here. 


\section{Supplementary material}

S1- RDG 525 is the best material for 3D printing

Considering the purpose of this system, one of the most important points was the possibility of platform sterilization, making it suitable for cell culture by autoclaving cycles. For the performance of cell migration tests, the flow system must be placed in an incubation chamber at $37^{\circ} \mathrm{C}$ and $5 \% \mathrm{CO} 2$ under humidity conditions for at least 24 hours and must support these temperatures without deformation. Under these conditions, the materials initially chosen were RGD 720 (general purpose) and RGD 525 (high temperature). Analyses of the pieces were carried out by coordinate measurement of the pieces before and after autoclaved cycles and finite-element simulation (Abaqus) (data not shown) to quantify the deformations of the material under the high-temperature conditions previously mentioned. It was possible to conclude that with the RGD 525 material, the small deformations induced by autoclaved cycles allowed the planned test setups to be performed and had no influence on the results; therefore, this was the best material for our assays.

S2- Scheme-summary of the conditions applied for the study of the different parameters analyzed throughout this article.

\section{\begin{tabular}{l|l|l} 
Analysis & Collagen concentration & Conditions
\end{tabular}}

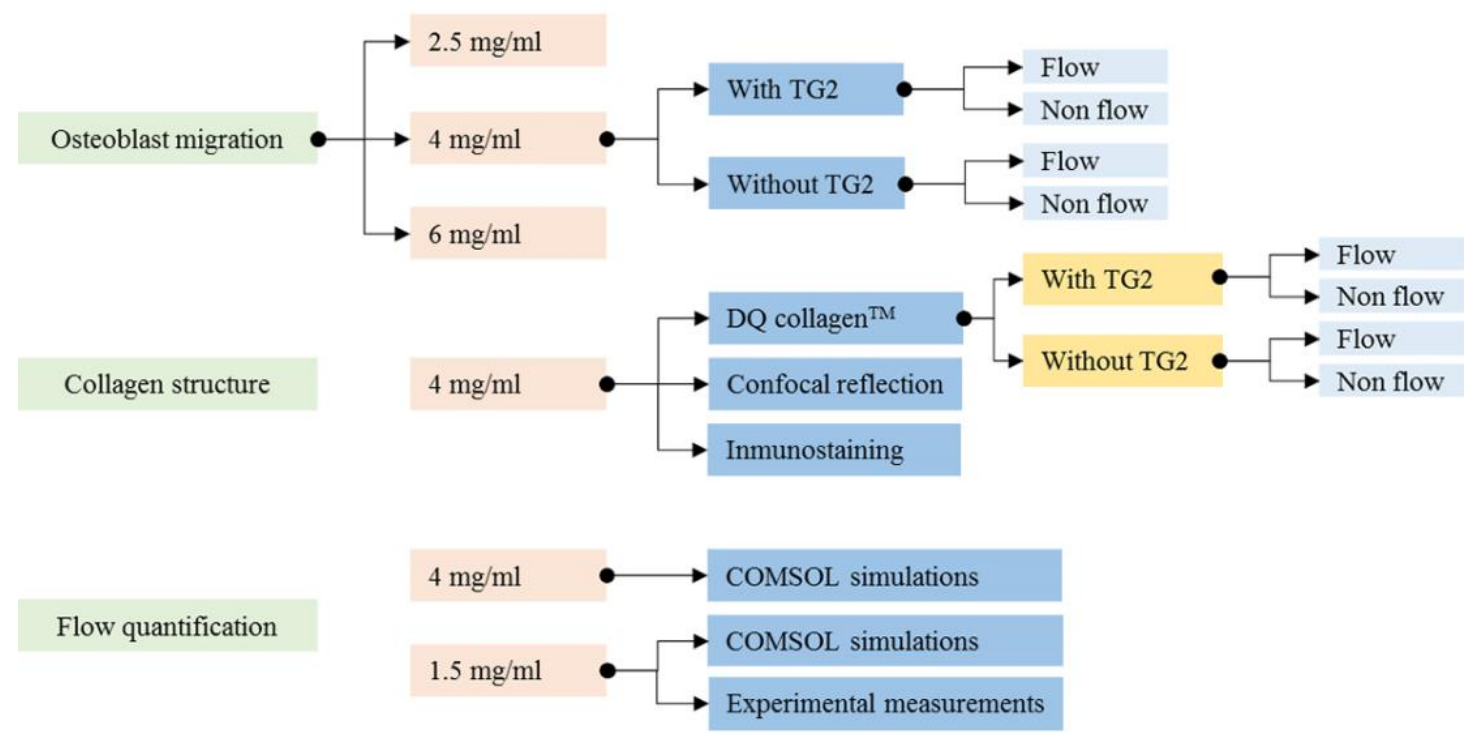

Figure S1. Flowchart of the conditions performed for the analysis of the different parameters studied in this work.

S3- Coincident fibers by using different methodologies of collagen matrix visualization

To evaluate the coincident position of collagen fibers in all of methodologies, an algorithm to automatically obtain the true colocalization proposed for Villalta et al. 2011 was executed in MATLAB [76]. Manders overlap coefficient (R) and Pearson's correlation coefficient (r) were calculated basing on the fluorescence intensity of each pixel in a pair of images.

This is the post-print version of the following article: Del Amo, C., Olivares, V., Cóndor, M., Blanco, A., Santolaria, J., Asín, J., Borau C \& García-Aznar, J. M. (2018). Matrix architecture plays a pivotal role in 3D osteoblast migration: The effect of interstitial fluid flow. Journal of the mechanical behavior of biomedical materials, 83, 52-62. doi: 10.1016/j.jmbbm.2018.04.007, which has been published in final form here. 
According to the positive, negative or null proportional contribution of pixel pairs, the correlation of images varied, being positively correlated when $r>0$, independently distributed when $r \approx 0$, or negatively correlated. As the algorithm used to analyze the images allows only two simultaneous comparisons, in the case of hydrogels with DQ-Collagen, all options were cross-checked between them (confocal reflection- DQCollagen fluorescence - Alexa 546 immunostaining). For the case of the gel without collagen DQ, only a comparison of images was required (see table S1). Figure S2 shows the results obtained in the analysis of coincident fibers for a pair of selected images to validate our novel method.

Based on the results from the exhaustive image and statistical analyses performed, our hypothesis of coincident collagen fibers in the case of DQ-Collagen application could be accepted. $r$ values fluctuated between 0.36 and 0.79 and were positive in all cases, which indicates a true correlation between the images.

In addition, to verify that the values obtained from the correlation analysis were not coincidental, the same test was performed, but the pixels of the image were distributed in a random manner. Statistical analysis was then carried out to check that the measured values of $\mathrm{R}$ and $\mathrm{r}$ were significantly higher than those measured when there was only random overlap.

\begin{tabular}{|c|c|c|c|c|}
\hline & Pearson & P value* & Manders Overlap & P Value* \\
\hline \multicolumn{5}{|c|}{ Collagen hydrogel } \\
\hline Alexa $546+$ C Reflection & 0.36752287 & $1 \mathrm{E}-16$ & 0.5310421 & $1 \mathrm{E}-16$ \\
\hline \multicolumn{5}{|c|}{ DQ Ccollagen hydrogel } \\
\hline Alexa 546+ DQ collagen & 0.79859203 & $1 \mathrm{E}-16$ & 0.8585667 & $1 \mathrm{E}-16$ \\
\hline Alexa $546+$ C Reflection & 0.40916434 & $1 \mathrm{E}-16$ & 0.58667082 & $1 \mathrm{E}-16$ \\
\hline DQ Collagen + C. Reflection & 0.44234043 & $1 \mathrm{E}-16$ & 0.60646546 & $1 \mathrm{E}-16$ \\
\hline
\end{tabular}

Table S1. Corresponding values for Manders overlap coefficient and Pearson's correlation. *P value of $0.95 \%$ indicates significant colocalization $(\mathrm{p}<0.05)$.

This is the post-print version of the following article: Del Amo, C., Olivares, V., Cóndor, M., Blanco, A., Santolaria, J., Asín, J., Borau C \& García-Aznar, J. M. (2018). Matrix architecture plays a pivotal role in 3D osteoblast migration: The effect of interstitial fluid flow. Journal of the mechanical behavior of biomedical materials, 83, 52-62. doi: 10.1016/j.jmbbm.2018.04.007, which has been published in final form here. 
A

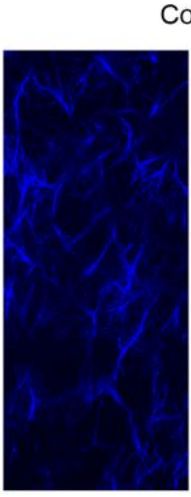

Confocal images
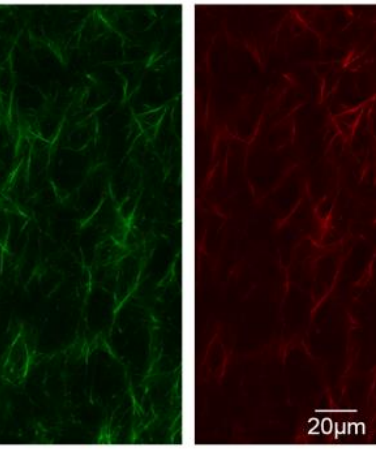

C

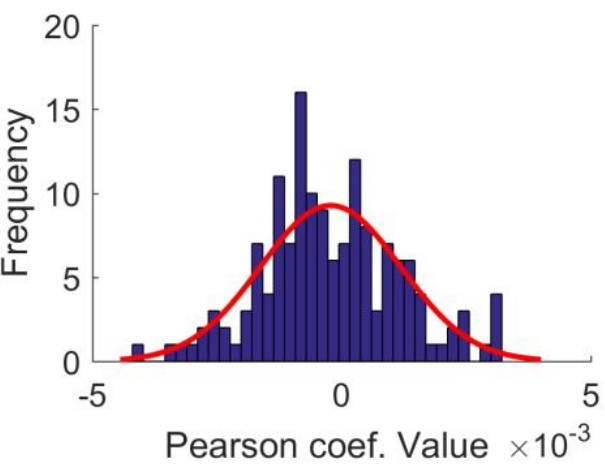

B Inmunostaining vs DQ-Collagen
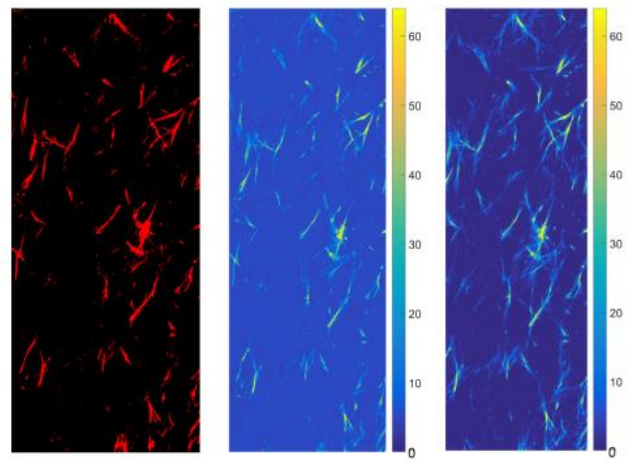

D

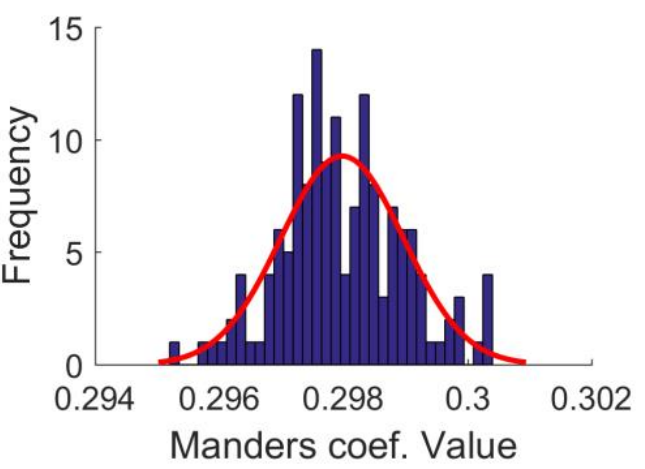

Figure S2. Confocal reflection of a collagen hydrogel, DQ-Collagen and the immunostained hydrogel images captured with a 60X oil objective are shown in A. B) Images from DQ-Collagen and immunostained collagen were compared, obtaining the colocalization mask followed by the Pearson and Manders map. Distribution of Pearson's correlation coefficient (C) and Manders overlap coefficient (D) for the fluorescence intensity of every pixel in a pair of images.

\section{S4- Similar flow rate estimation by different methodologies}

To simplify the quantification of fluid flow into the hydrogel, a $1.5 \mathrm{mg} / \mathrm{ml}$ collagen concentration was selected for this experiment. The collagen network for this concentration presented a disposition of the fiber network that facilitated the visualization of beads inside the hydrogel. The results obtained by particle tracking and computational simulations were similar. These results allowed us to determine how fluid flow was distributed along the chambers and to extrapolate distribution for the $4 \mathrm{mg} / \mathrm{ml}$ hydrogel. Previously, the permeability of both hydrogels was determined, and the following values were obtained: $3.19 \cdot 10 \mathrm{E}-13$ $\mathrm{m}^{2}$ for the $1.5 \mathrm{mg} / \mathrm{ml}$ hydrogel and $3.82 \cdot 10 \mathrm{E}-13$ for the $4 \mathrm{mg} / \mathrm{ml}$ hydrogel. These data were incorporated into a COMSOL model, and the velocities for a $4 \mathrm{mg} / \mathrm{ml}$ gel medium $(23.47 \pm 0.7714 \mu \mathrm{m} / \mathrm{s})$ and for a 1.5 $\mathrm{mg} / \mathrm{ml}$ gel medium $(24.238 \pm 1.0255 \mu \mathrm{m} / \mathrm{s})$ were obtained. Data for each condition were taken from the area between the two posts and across the width of the gel, including the central and lateral areas of the gel (the results are summarized in table $\mathrm{S} 2$ ).

\begin{tabular}{|lc|}
\hline Velocity $1.5 \mathrm{mg} / \mathrm{ml}$ Collagen $(\mu \mathrm{m} / \mathrm{s})$ & Velocity $4 \mathrm{mg} / \mathrm{ml}$ \\
& Collagen $(\mu \mathrm{m} / \mathrm{s})$ \\
\hline
\end{tabular}

This is the post-print version of the following article: Del Amo, C., Olivares, V., Cóndor, M., Blanco, A., Santolaria, J., Asín, J., Borau C \& García-Aznar, J. M. (2018). Matrix architecture plays a pivotal role in 3D osteoblast migration: The effect of interstitial fluid flow. Journal of the mechanical behavior of biomedical materials, 83, 52-62. doi: 10.1016/j.jmbbm.2018.04.007, which has been published in final form here. 


\begin{tabular}{|c|c|c|}
\hline Particles tracking & \multicolumn{2}{|c|}{ COMSOL Multiphysics 4.3} \\
\hline $26.0655 \pm 15.6797$ & $26.86 \pm 4.22$ & $25.2453 \pm 8.1634$ \\
\hline
\end{tabular}

Table S2. Values of fluid speed inside the $1.5 \mathrm{mg} / \mathrm{ml}$ and $4 \mathrm{mg} / \mathrm{ml}$ collagen hydrogels.

Another flow rate applied to the osteoblast culture was $0.1 \mu 1 / \mathrm{min}$, and $2.347 \mu \mathrm{m} / \mathrm{s}$ was the corresponding value estimated for this case.

It is worth noting the high dispersion obtained (comparable to the mean). This effect might be caused by the high concentration of particles that were retained in the meniscus of the gel versus the long distance traveled by other groups of beads (Figure S3, A and B)

On the other hand, the computational model showed a symmetric distribution of fluid inside the chamber when analyzing the streamline trajectories (Figure S3 C and D).
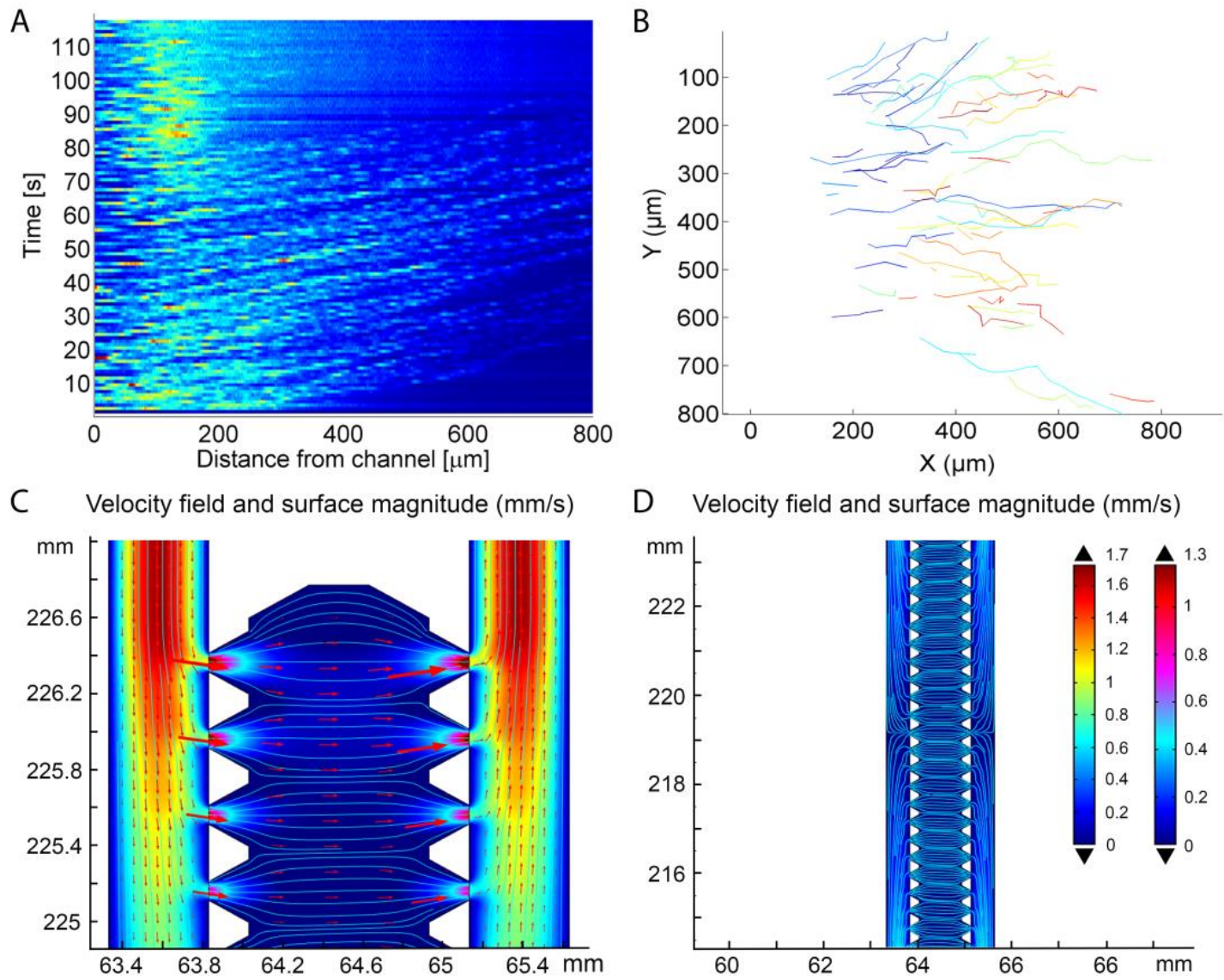

Figure S3. A and B: Quantification of bead movement inside the collagen hydrogel and its trajectories in the substracted (A) and merged (B) images. C and D: Estimation of fluid flow inside the chamber. COMSOL simulation of the upper chip area $(C)$ and the central zone of the chip (D). Streamlines are represented in light blue, and the flow direction is indicated by the red arrows.

This is the post-print version of the following article: Del Amo, C., Olivares, V., Cóndor, M., Blanco, A., Santolaria, J., Asín, J., Borau C \& García-Aznar, J. M. (2018). Matrix architecture plays a pivotal role in 3D osteoblast migration: The effect of interstitial fluid flow. Journal of the mechanical behavior of biomedical materials, 83, 52-62. doi: 10.1016/j.jmbbm.2018.04.007, which has been published in final form here. 


\begin{tabular}{|lc|c|c|c|}
\cline { 2 - 5 } \multicolumn{1}{c|}{} & Q50 & Q75 & Q90 & Q95 \\
\hline Collagen & 1.88 & 2.66 & 3.43 & 4.86 \\
\hline $\begin{array}{l}\text { Collagen + } \\
\mathbf{1} \boldsymbol{\mu l} / \mathbf{m i n} \text { FF }\end{array}$ & 1.64 & 2.32 & 2.99 & 4.23 \\
\hline Collagen + TG2 & 1.59 & 2.25 & 2.9 & 4.1 \\
\hline $\begin{array}{l}\text { Collagen + } \\
\mathbf{1} \mathbf{l} / \mathbf{m i n} \text { FF+ TG2 }\end{array}$ & 1.59 & 2.25 & 2.9 & 4.11 \\
\hline
\end{tabular}

Table S3. From the fitted distribution of data, the average of the higher quantiles for each condition was calculated and is shown in this table.

\section{S5- Effect of collagen concentration on osteoblast migration}

In order to analyze osteoblast migration in static conditions in three different collagen concentrations, new independent experiments were performed. The selected collagen concentrations were: $2.5 \mathrm{mg} / \mathrm{ml}, 4 \mathrm{mg} / \mathrm{ml}$ and $6 \mathrm{mg} / \mathrm{ml}$. The preparation of collagen hydrogel was performed by following the protocol described in the section 2.2 and using the same cell concentration.

Results show statistically significant differences in osteoblast speed migration in $2.5 \mathrm{mg} / \mathrm{ml}$ gels compared to 4 and $6 \mathrm{mg} / \mathrm{ml}$ gels, especially regarding effective velocities. In the first case, due to the properties of hydrogel, which has a higher pore size and lower stiffness, the migration of the cells was faster and more directed than in the other conditions. With the increase in the concentration of collagen, and therefore in the stiffness of the matrix, the migration speeds (Figure S4A) followed by the cells (see Figure S4B) dropped, and the trajectories were shorter and confined to a smaller region.

A

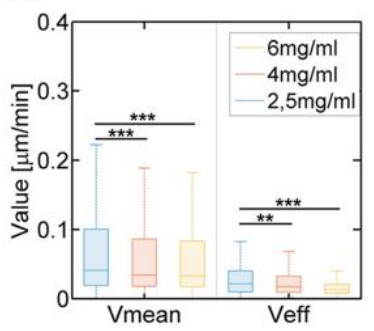

B

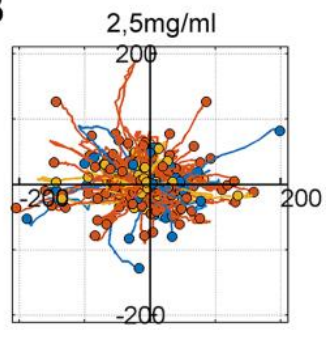

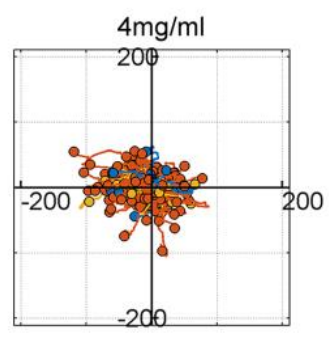

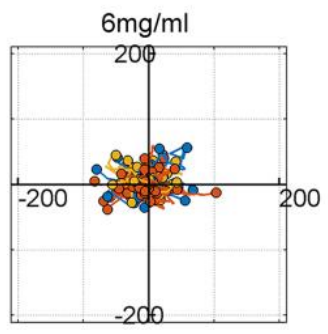

Figure S4. A) Mean and effective speeds of HOB cells cultured in $2.5 \mathrm{mg} / \mathrm{ml}, 4 \mathrm{mg} / \mathrm{ml}$ and $6 \mathrm{mg} / \mathrm{ml} \mathrm{collagen}$ hydrogels. Anova tests were performed for statistical significance $* * * p<0,001 ; * * p<0,01 ;{ }^{*} p<0,05$. B) Cell trajectories for the different conditions of collagen concentrations.

This is the post-print version of the following article: Del Amo, C., Olivares, V., Cóndor, M., Blanco, A., Santolaria, J., Asín, J., Borau C \& García-Aznar, J. M. (2018). Matrix architecture plays a pivotal role in 3D osteoblast migration: The effect of interstitial fluid flow. Journal of the mechanical behavior of biomedical materials, 83, 52-62. doi: 10.1016/j.jmbbm.2018.04.007, which has been published in final form here. 


\section{Figures}

Figure 1. A) The general conformation of the microfluidic device and its transversal section to visualize the distribution of channels. B) A representative diagram of the fluid flow setup (1: input and output connectors; 2: microscopic visualization area; 3 : screw holes for the locking system; 4: recess for microfluidic chip insertion).

Figure 2. A) Stack of confocal cross-sections. B) Binarized stack visualized with Paraview. C) Ct-Fire reconstruction of the collagen network viewed in MATLAB. D) 2D projection of the obtained skeleton of a collagen matrix.

Figure 3. A) Reference semi-sphere with numeration of the divisions used to plot the spatial fiber orientation. B) 3D rose diagrams of fiber orientation for the different conditions. C) Percentage of fibers in the corresponding sector for each condition

Figure 4. A) Mean and effective speed of HOB cells. Each condition comprises 3 repetitions ( 60 cells analyzed per repetition). Anova tests were performed for statistical significance. ${ }^{* * *} \mathrm{p}<0.001 ;{ }^{* *} \mathrm{p}<0.01$; $* \mathrm{p}<0.05$. B) Directionality ratio over time for each condition.

Figure 5. Trajectories of cells whose starting point was centered on the coordinate origin. The conditions analyzed were collagen or collagen + TG2 in the presence or absence of fluid flow, and the cells embedded in the matrix were altered with $1 \mu \mathrm{l} / \mathrm{min}$ of fluid flow, presenting higher directionality.

Figure 6. A) Examples of cell morphologies in the different mechanical conditions: Collagen, Collagen + $1 \mu \mathrm{l} / \mathrm{min}$ flow, Collagen $+\mathrm{TG} 2$, and Collagen + TG2 $+1 \mu \mathrm{l} / \mathrm{min}$ flow. Samples were fixed; stained for vinculin (green), phalloidin (actin, red) and DAPI (nuclei, blue); and imaged using an Olympus Fluoview FV10i confocal microscope with a 60XW objective. B) Cell major axis length. C) Cell solidity. D) An example of a cell processed with the MATLAB script (image captured using a Nikon D-Eclipse C1 confocal microscope with a $40 \mathrm{X}$ oil objective). $\mathrm{N} \geq 20$ cells analyzed for each condition. Anova tests were performed for statistical significance. ${ }^{* * *} \mathrm{p}<0.001 ;{ }^{* *} \mathrm{p}<0.01 ;{ }^{*} \mathrm{p}<0.05$.

Figure S1. Flowchart of the conditions performed for the analysis of the different parameters studied in this work.

Figure S2. Confocal reflection of a collagen hydrogel, DQ-Collagen and the immunostained hydrogel images captured with a 60X oil objective are shown in A. B) Images from DQ-Collagen and immunostained collagen were compared, obtaining the colocalization mask followed by the Pearson and Manders map. Distribution of Pearson's correlation coefficient (C) and Manders overlap coefficient (D) for the fluorescence intensity of every pixel in a pair of images.

Figure S3. A and B: Quantification of bead movement inside the collagen hydrogel and its trajectories in the substracted (A) and merged (B) images. C and D: Estimation of fluid flow inside the chamber. COMSOL simulation of the upper chip area (C) and the central zone of the chip (D). Streamlines are represented in light blue, and the flow direction is indicated by the red arrows.

Figure S4. A) Mean and effective speed of HOB cells cultured in $2.5 \mathrm{mg} / \mathrm{ml}, 4 \mathrm{mg} / \mathrm{ml}$ and $6 \mathrm{mg} / \mathrm{ml}$ collagen hydrogels. Anova tests were performed for statistical significance ${ }^{* * *} \mathrm{p}<0,001 ; * * \mathrm{p}<0,01 ;{ }^{*} \mathrm{p}<0,05$. B) Cell trajectories for the different conditions of collagen concentrations

This is the post-print version of the following article: Del Amo, C., Olivares, V., Cóndor, M., Blanco, A., Santolaria, J., Asín, J., Borau C \& García-Aznar, J. M. (2018). Matrix architecture plays a pivotal role in 3D osteoblast migration: The effect of interstitial fluid flow. Journal of the mechanical behavior of biomedical materials, 83, 52-62. doi: 10.1016/j.jmbbm.2018.04.007, which has been published in final form here. 


\section{Tables}

Table 1. Mechanical properties of the collagen hydrogels used for the in vitro experiments. Three samples were analyzed for the cases without TG2 and $25 \mu \mathrm{g} / \mathrm{ml}$ of TG2. Data taken with permission from [35].

Table 2. Values of porosity and pore size quantified from the matrix architecture and fiber distribution analyses.

Table 3. Values obtained in the cell tracking measurements of the mean and effective velocity. Each condition comprises 3 repetitions ( $\sim 60$ cells analyzed per repetition). Anova tests were performed for statistical significance. ${ }^{* * *} \mathrm{p}<0.001 ; * * \mathrm{p}<0.01 ;{ }^{*} \mathrm{p}<0.05$.

Table 4. Diffusivity coefficient of each condition and exponential adjustment parameter (alpha) obtained from the minimum square displacement (MSD) curve fitting $[35,56]$. Each condition comprises 3 repetitions ( $\sim 60$ cells analyzed per repetition). T-tests were performed against the null hypothesis that $\alpha=$ 1 (purely diffusive motion) ${ }^{* * *} \mathrm{p}<0.001 ; * * \mathrm{p}<0.01 ;{ }^{*} \mathrm{p}<0.05$.

Table S1. Corresponding values for Manders overlap coefficient and Pearson's correlation. *P value of $0.95 \%$ indicates significant colocalization $(\mathrm{p}<0.05)$.

Table S2. Values of fluid speed inside the $1.5 \mathrm{mg} / \mathrm{ml}$ and $4 \mathrm{mg} / \mathrm{ml}$ collagen hydrogels.

Table S3. From the fitted distribution of data, the average of the higher quantiles for each condition was calculated and is shown in this table.

This is the post-print version of the following article: Del Amo, C., Olivares, V., Cóndor, M., Blanco, A., Santolaria, J., Asín, J., Borau C \& García-Aznar, J. M. (2018). Matrix architecture plays a pivotal role in 3D osteoblast migration: The effect of interstitial fluid flow. Journal of the mechanical behavior of biomedical materials, 83, 52-62. doi: 10.1016/j.jmbbm.2018.04.007, which has been published in final form here. 